\title{
The vesicular release probability sets the strength of individual Schaffer collateral synapses
}

\section{Céline Dürst}

University Medical Center Hamburg-Eppendorf

\section{J. Simon Wiegert}

University Medical Center Hamburg-Eppendorf https://orcid.org/0000-0003-0893-9349

\section{Christian Schulze}

University Medical Center Hamburg-Eppendorf

\section{Nordine Helassa}

St George's, University of London https://orcid.org/0000-0003-3743-1886

\section{Katalin Torok}

St George's, University of London https://orcid.org/0000-0002-3107-4534

Thomas Oertner ( $\nabla$ thomas.oertner@zmnh.uni-hamburg.de)

University Medical Center Hamburg-Eppendorf https://orcid.org/0000-0002-2312-7528

\section{Article}

Keywords: Schaffer collateral synapses, vesicular release, neurotransmitters

Posted Date: October 14th, 2020

DOI: https://doi.org/10.21203/rs.3.rs-89676/v1

License: (c) (i) This work is licensed under a Creative Commons Attribution 4.0 International License. Read Full License

Version of Record: A version of this preprint was published at Nature Communications on October 17th, 2022. See the published version at https://doi.org/10.1038/s41467-022-33565-6. 


\title{
The vesicular release probability sets the strength of individual Schaffer collateral synapses
}

\author{
Céline D. Dürst ${ }^{1}$, J. Simon Wiegert ${ }^{1,2}$, Christian Schulze ${ }^{1}$, Nordine Helassa ${ }^{3,4}$, Katalin \\ Török ${ }^{3}$ and Thomas G. Oertner ${ }^{1 *}$
}

\author{
${ }^{1}$ Institute for Synaptic Physiology, Center for Molecular Neurobiology Hamburg (ZMNH), 20251 \\ Hamburg, Germany \\ ${ }^{2}$ Research Group Synaptic Wiring and Information Processing, Center for Molecular \\ Neurobiology Hamburg (ZMNH), 20251 Hamburg, Germany \\ ${ }^{3}$ Cell Biology and Genetics Research Centre, Molecular and Clinical Sciences Research \\ Institute, St George's, University of London, London SW17 ORE, UK \\ ${ }^{4}$ Department of Cardiovascular and Metabolic Medicine, Institute of Life Course and Medical \\ Sciences, University of Liverpool, Liverpool, L69 3BX
}

*Correspondence: thomas.oertner@zmnh.uni-hamburg.de

\begin{abstract}
Information processing in the brain is controlled by quantal release of neurotransmitters, a tightly regulated process. Even in a single axon, presynaptic boutons differ in the number of docked vesicles, but it is not known if the vesicular release probability ( $\left.p_{\text {ves }}\right)$ is homogenous or variable between individual boutons. We optically measured evoked transmitter release at individual Schaffer collateral synapses using the genetically encoded glutamate sensor iGluSnFR, localizing the fusion site on the bouton with high spatiotemporal precision. Fitting a binomial model to measured response amplitude distributions allowed us to extract the quantal parameters $N, p_{\text {ves, }}$ and $q$. Schaffer collateral boutons typically released only a single vesicle under low $p_{\text {ves }}$ conditions and switched to multivesicular release in high calcium saline. We found that $p_{\text {ves }}$ was highly variable between individual boutons and had a dominant impact on presynaptic output.
\end{abstract}




\section{Introduction}

2 The conceptual framework of quantal synaptic transmission was developed at the frog

3 neuromuscular junction (NMJ), a giant synapse containing thousands of transmitter vesicles ${ }^{1}$.

4 Statistical analysis of electrophysiological recordings from innervated muscle cells suggested

5 that neurotransmitter is released in multi-molecular packets ('quanta') which were later identified

6 as synaptic vesicles. The strength of a specific NMJ could be mathematically described ${ }^{2}$ as the

7 product of the number of release sites $N$, their probability of release $p_{\text {ves, }}$ and the postsynaptic

8 response to the release of a single quantum, $q$. Applying quantal analysis to the very small

9 synapses of the mammalian brain is not straightforward. In the brain, each neuron receives

10 thousands of synapses, each of which has a different electrotonic distance from the postsynaptic

11 cell body. Viewed from the soma, there is no unitary response: A single vesicle released at a

12 distal dendritic synapse creates a much smaller EPSP than a vesicle released at a perisomatic

13 synapse, making classical electrophysiological quantal analysis impossible. Attempts to

14 electrically stimulate individual synapses gave rise to the hypothesis that individual synapses in

15 the central nervous system can only release a single vesicle ('uniquantal release')2. With

16 electrophysiology alone, however, it is difficult to distinguish between stimulation of multiple

17 synapses and stimulation of a single synapse that is capable of multivesicular release.

18 Optical methods based on fluorescent calcium indicators have been used to resolve excitatory

19 postsynaptic calcium transients (EPSCaTs) at individual synapses. Under conditions of high

20 release probability, larger EPSCaTs were observed ${ }^{3}$. However, the amplitude of spine calcium

21 transients depends on the local membrane potential in a highly non-linear fashion. The low

22 number and stochastic behavior of postsynaptic NMDA receptors ${ }^{4}$ adds variability to EPSCaTs,

23 making it difficult to draw conclusions about vesicular release statistics from EPSCaT amplitude

24 distributions. Furthermore, experimental manipulation of divalent ion concentration $\left(\mathrm{Ca}^{2+}, \mathrm{Mg}^{2+}\right.$,

$25 \mathrm{Sr}^{2+}$ ) affect both the release machinery and the response of the optical calcium sensor, making

26 the results of ion-substitution experiments difficult to interpret. In dissociated neuronal culture, it

27 is possible to monitor vesicle fusion events using $\mathrm{pH}$-sensitive green fluorescent proteins

28 (pHluorins $)^{5,6}$. A limitation of $\mathrm{pH}$-based fusion detection is the lack of information about the filling

29 state of the released vesicles ${ }^{7}$.

30 Here we use the genetically encoded glutamate sensor iGluSnFR ${ }^{8}$ to measure glutamate

31 concentrations in the synaptic cleft ${ }^{9,10}$. We show that this probe is sensitive enough to detect the

32 fusion of single vesicles at Schaffer collateral boutons in organotypic hippocampal cultures. At

33 near-physiological calcium concentrations ${ }^{11}$, synapses produced mostly failures and released

34 single vesicles. Elevating the extracellular $\mathrm{Ca}^{2+}$ concentration caused synapses to increase their 
35 release probability and to release multiple vesicles in response to single action potentials (APs).

36 By localizing the fusion site on the surface of the presynaptic bouton with high precision, we

37 show that multivesicular release occurs in a confined area, the active zone, which is stable over

38 time. Using dual patch-clamp recordings and Monte Carlo simulations of glutamate diffusion, we

39 show that the dynamic range of iGluSnFR is similar to postsynaptic AMPA receptors, although

40 the kinetics of the underlying glutamate transients in the synaptic cleft is an order of magnitude

41 faster. Thus, iGluSnFR signals are a good proxy for postsynaptic responses, but do not report

42 the speed of glutamate diffusion out of the synaptic cleft. Comparing quantal parameters across

43 boutons, we show that $p_{\text {ves }}$ is the main determinant of synaptic strength under low release

44 probability conditions, whereas $N$ limits the strength of a given synapse under conditions of high

45 release probability.

\section{Results}

47 We transfected individual CA3 pyramidal neurons in organotypic slices of rat hippocampus with

48 iGluSnFR and the red fluorescent protein tdimer2 (Fig. 1a-b). Two to four days after transfection,

49 we transferred the cultures to the recording chamber of a two-photon microscope (Extended

50 Data Fig.1). As iGluSnFR is relatively dim in the absence of glutamate, we used the red

51 cytoplasmic fluorescence to visualize soma, axons and boutons of transfected CA3 pyramidal

52 cells (Fig. 1c-d). To evoke and measure release, we patch-clamped the transfected cells and

53 triggered single APs by brief somatic current injections while imaging bouton fluorescence in

54 CA1 stratum radiatum (Fig. 1e). Boutons belonging to the patched CA3 neuron were easily

55 identified by a rapid increase in green fluorescence milliseconds after the somatically-triggered

56 AP (Fig. 1f). Recording temperature was stabilized at $33 \pm 1^{\circ} \mathrm{C}$ by Peltier-heating the oil

57 immersion condenser.

58 The fast rise and decay kinetics of iGluSnFR ${ }^{8,12}$ made it challenging to capture the peak of

59 iGluSnFR fluorescence transients using the relatively slow raster scanning mode. Straight line

60 scans across an individual bouton provide much better temporal resolution (up to $1 \mathrm{kHz}$ ), but are

61 extremely sensitive to mechanical drift of the tissue: If the scan line misses the center of the

62 rapidly diffusing cloud of glutamate, the true amplitude of individual release events will be

63 underestimated. To capture the peak of the iGluSnFR signal, we modified Scanlmage $3.8^{13}$ to

64 allow user-defined spiral scans at $500 \mathrm{~Hz}$, thus sampling the entire surface of the bouton every

65 two milliseconds (Fig. 1g, left). As we did not know the exact location of the fusion site a priori,

66 we did not use a static region of interest (ROI), but evaluated the spatial positions 
a

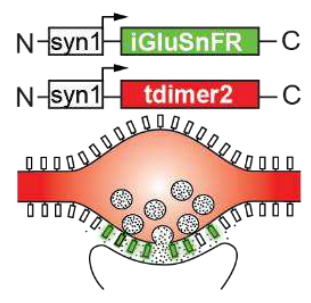

b

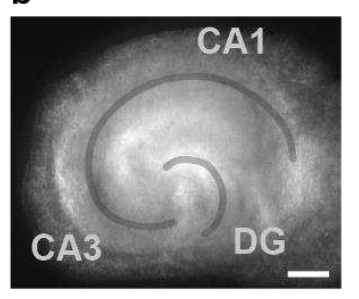

C

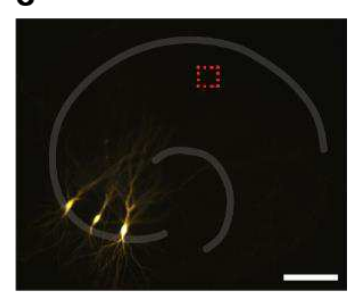

d

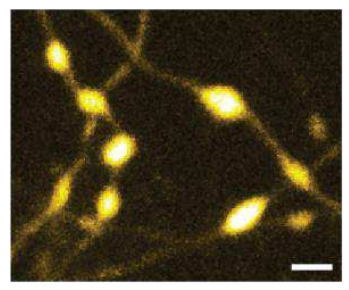

e
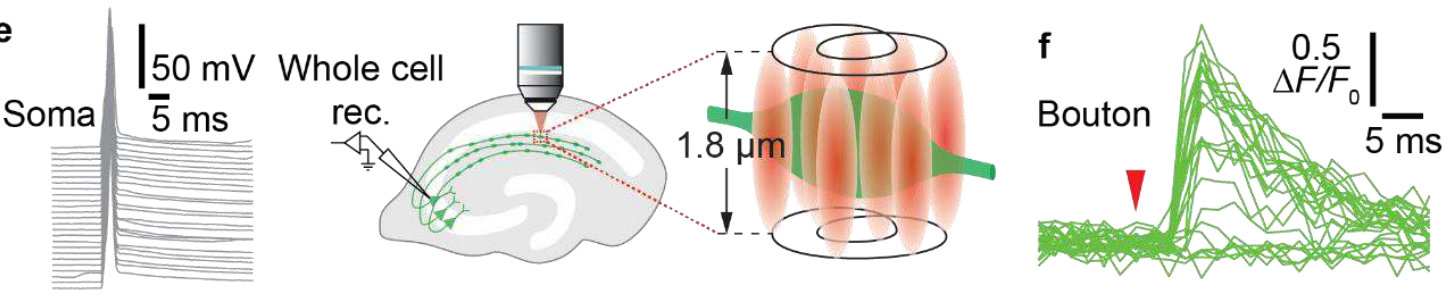

9
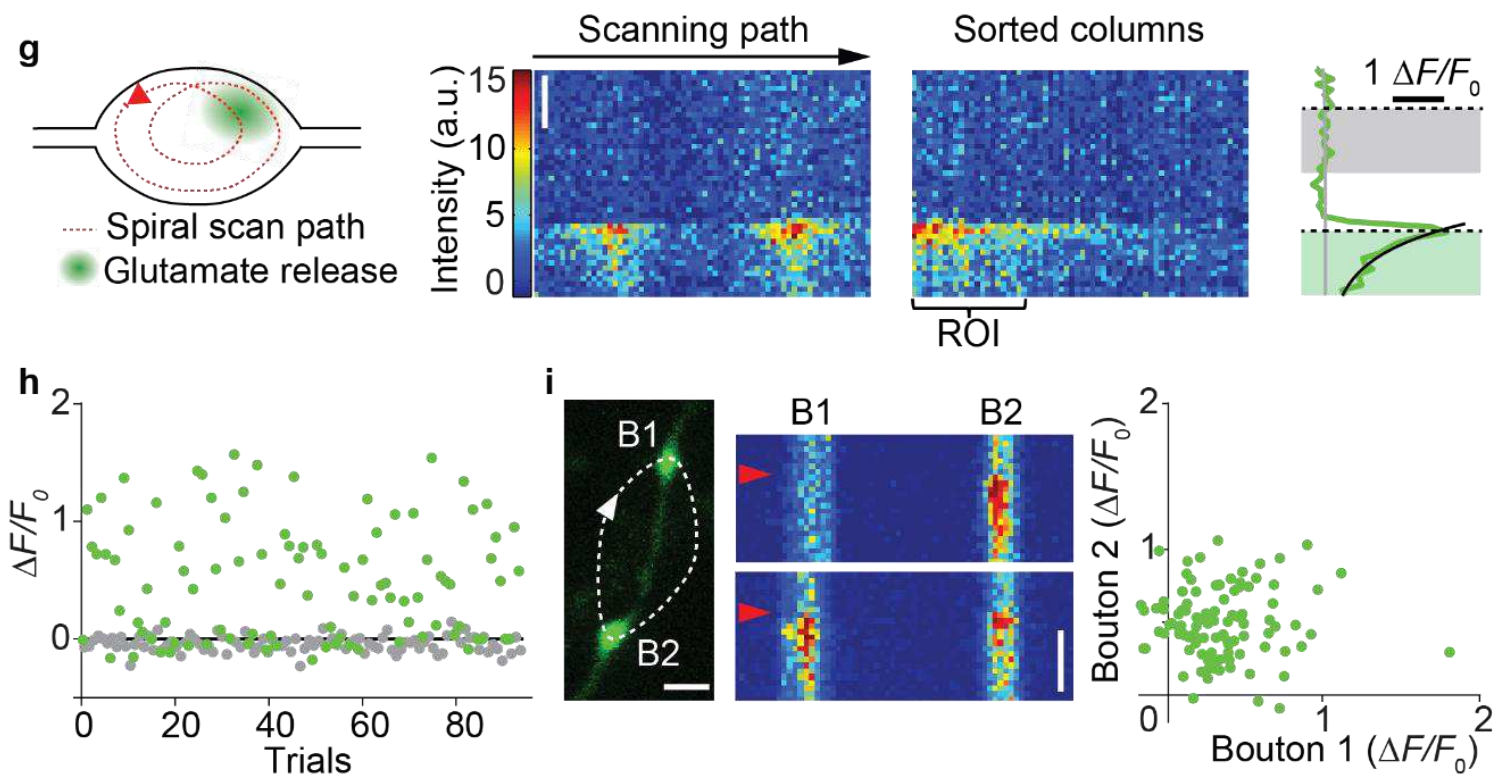

68
Figure 1: iGluSnFR expression in CA3 pyramidal cells of organotypic slice culture of rat hippocampus.

a) En passant bouton co-expressing the cytoplasmic red fluorescent protein tdimer2 and green iGluSnFR exposed to the extracellular space. b) Transmitted light image of transfected organotypic hippocampal culture. Scale bar, $500 \mu \mathrm{m}$. c) Fluorescence image of three transfected CA3 neurons. Area for synaptic imaging is indicated (dotted box). Scale bar, $500 \mu \mathrm{m}$. d) Maximum intensity projection of a two-photon image stack of CA3 axons and their en passant boutons co-expressing tdimer2 and iGluSnFR in CA1 stratum radiatum. Scale bar, $1 \mu \mathrm{m}$. e) APs were reliably elicited in a transfected neuron by somatic current injections while glutamate release was simultaneously imaged from a single Schaffer collateral bouton in CA1. Owing to the elongated point-spread function (PSF; red oval objects), the upper and lower surface of a bouton were simultaneously sampled by $500 \mathrm{~Hz}$ spiral scanning. f) Optical recording (iGluSnFR fluorescence) from a single Schaffer collateral bouton in CA1, showing a broad distribution of amplitudes and occasional failures. Red arrowhead indicates somatic current injection in CA3. g) Release events were typically sampled twice during every spiral scan. Fluorescence intensity (single trial) coded in rainbow colors. At $t=58 \mathrm{~ms}$, a glutamate release event occurred. Scale bar, $20 \mathrm{~ms}$. Only columns with $\Delta F>1 / 2 \max (\Delta F)$ were analyzed (dynamic region-of-interest). Response amplitude was estimated by exponential fit (green area, black fit) normalized by the resting fluorescence of the bouton (gray area, grey fit). The intersection between the horizontal black dashed lines and the fits corresponds to the extracted amplitudes (average peak amplitude for each experiment). h) Response amplitude (green circles) was constant over time. A time window before stimulation was analyzed to estimate imaging noise (gray circles). Response amplitudes were monitored in ACSF containing $2 \mathrm{mM}\left[\mathrm{Ca}^{2+}\right]_{\mathrm{e}}$ at $33^{\circ} \mathrm{C}$. i) Monitoring two neighboring boutons located on the same axon. Scale bar, $1 \mu \mathrm{m}$. Two trials showing independent release events. Red arrowhead indicates the stimulation onset. Scale bar, $20 \mathrm{~ms}$. iGluSnFR transients between neighboring boutons were not correlated $\left(R^{2}<0.0005\right)$. 
(columns in the time-space diagram) with the highest change in fluorescence (dynamic ROI,

Fig. 1g). If no clear stimulus-evoked change in fluorescence was detected (potential failure trial), the same columns as in the last trial were evaluated. In addition, we developed an automatic 3D repositioning routine to compensate for slow drift of the tissue, allowing stable optical recordings over hundreds of trials. To extract the amplitude of individual trials, we constructed a template (exponential decay) from several manually selected large responses. A single parameter (amplitude) was fit to match the template to each individual trial. Responses that exceeded $2 \sigma$ of baseline fluctuations were classified as successes. Occasionally, we observed green fluorescent particles moving through the axon. Such events were detected by their elevated $F_{0}$ at baseline and excluded from further analysis ( $2-5 \%$ of trials). At most boutons, the failure rate was stable over the time of the experiment (Fig. 1h). In principle, failure of glutamate release could be due to the stochastic nature of vesicle release or due to stochastic failures of AP propagation into individual branches of the extensive network of axon collaterals. In simultaneously imaged neighboring boutons, failures were not correlated, arguing for stochastic glutamate release (Fig. 1i).

Modulating synaptic release probability affects cleft glutamate concentration

107 Since presynaptic vesicle fusion is $\mathrm{Ca}^{2+}$-dependent, we expected a steep dependence of the synaptic release probability $p_{\text {syn }}$ on the extracellular $\mathrm{Ca}^{2+}$ concentration, $\left[\mathrm{Ca}^{2+}\right]_{\text {e. }}$ Indeed, switching $\left[\mathrm{Ca}^{2+}\right]_{\mathrm{e}}$ from $1 \mathrm{mM}$ to $4 \mathrm{mM}$ dramatically increased $p_{\text {syn }}$ from 0.26 to 0.87 (Fig. 2a and

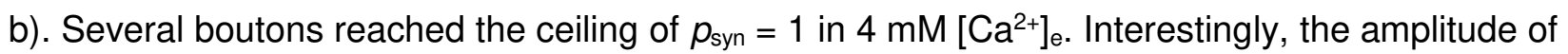
iGluSnFR signals (successes) increased as well from 0.84 to $1.76 \Delta F / F_{0}$ (Fig. 2b), indicating higher glutamate concentrations in the synaptic cleft under high $p_{\text {syn }}$ conditions. In low $\left[\mathrm{Ca}^{2+}\right]_{\text {e, }}$

113 success amplitudes were similar across boutons (Fig. 2c). In high $\left[\mathrm{Ca}^{2+}\right]_{\mathrm{e}}$, however, the same set 114 of boutons had variable success amplitudes that were strongly correlated with $p_{\text {syn. }}$. To further 115 explore the non-linear relationship between $p_{\text {syn }}$ and cleft glutamate concentrations, we

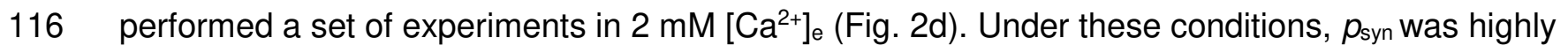
117 variable between individual boutons. Again, low $p_{\text {syn }}$ boutons produced consistent success 118 amplitudes $\left(58 \%\right.$ to $89 \% \Delta F / F_{0}$ for $\left.p_{\text {syn }}<0.5\right)$ while high $p_{\text {syn }}$ boutons produced considerably 119 larger successes. Boutons located next to each other on the same axon often had similar 120 properties (Extended Data Fig. 2).

121 Taken together, these data indicate that $p_{\text {syn }}$ is very variable between boutons and highly 122 dependent on $\left[\mathrm{Ca}^{2+}\right]_{\text {e }}$. Furthermore, as $p_{\text {syn }}$ increased, so did the cleft glutamate, consistent with 123 the capacity of Schaffer collateral boutons for multivesicular release ${ }^{14-19}$. 
a - Success oFailures Baseline

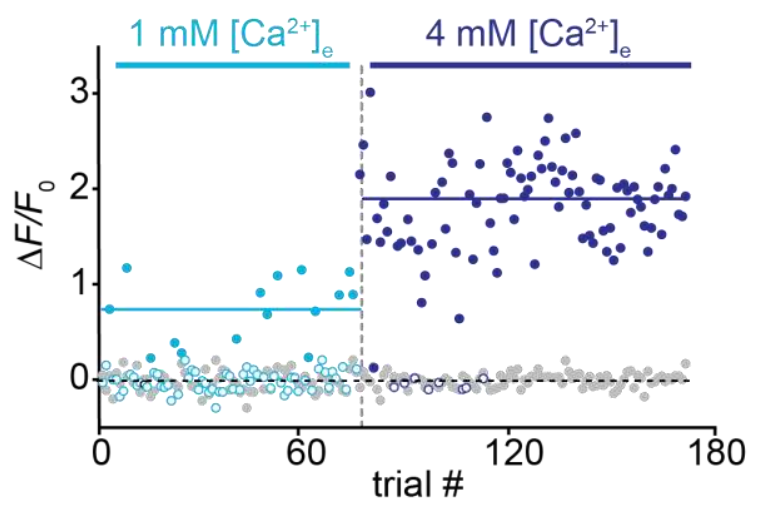

C

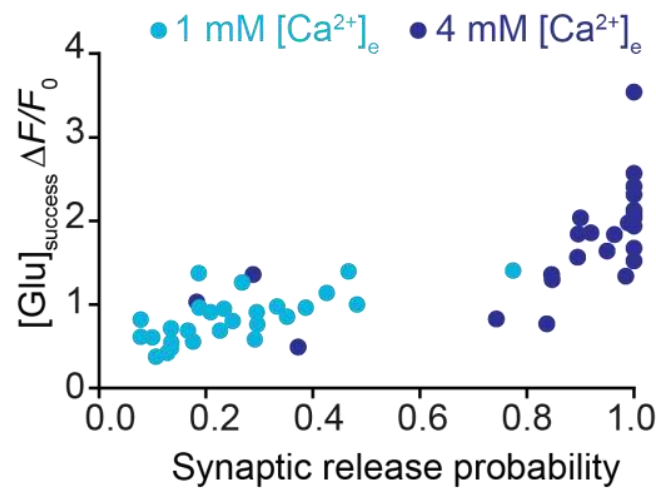

b
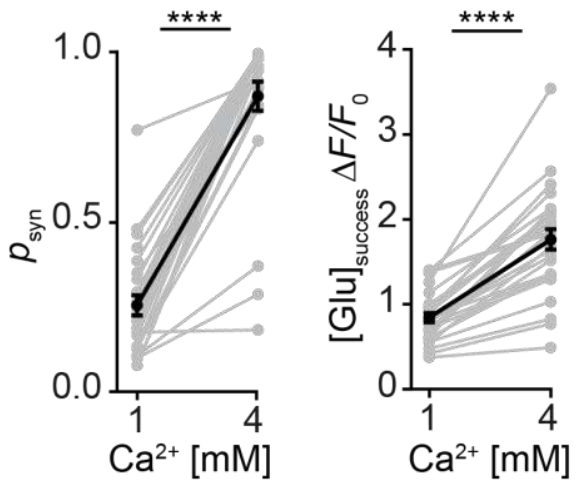

d

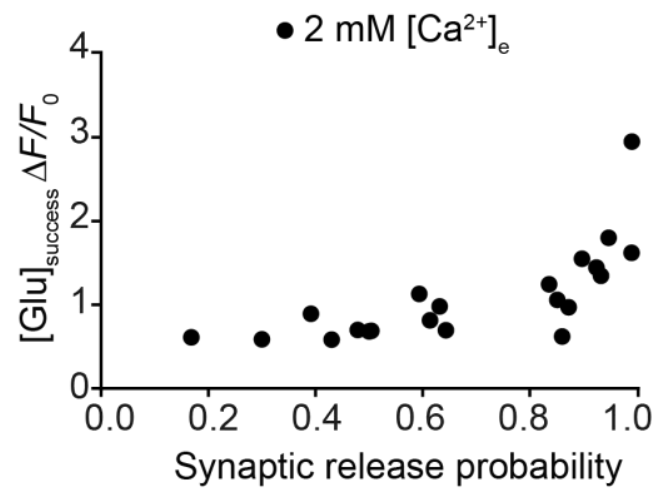

Figure 2: Increasing release probability increases the glutamate concentration of synaptic transmission events. a) Glutamate transients in a single bouton, switching from ACSF containing $1 \mathrm{mM}\left[\mathrm{Ca}^{2+}\right]_{\mathrm{e}}$ to $\left.4 \mathrm{mM}^{\mathrm{m}} \mathrm{Ca}^{2+}\right]_{\mathrm{e}}$. The light blue and the dark blue lines represent the average of the amplitude of successes in $\left.1 \mathrm{mM}^{[\mathrm{Ca}}{ }^{2+}\right]_{\mathrm{e}}$ and $4 \mathrm{mM}$ $\left[\mathrm{Ca}^{2+}\right]_{\mathrm{e}}$ respectively. b) Summary of all experiments in $1 \mathrm{mM} / 4 \mathrm{mM}\left[\mathrm{Ca}^{2+}\right]_{\mathrm{e}}$. The probability of successful glutamate

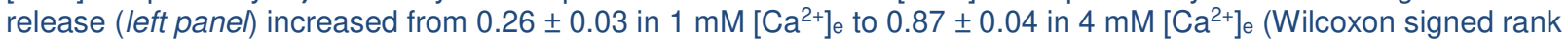
test, $p<0.0001, n=27$ boutons in 23 slices). The amplitude of fluorescence transients in trials classified as 'success' (right panel) increased from $0.84 \pm 0.056 \Delta F / F_{0}$ in $1 \mathrm{mM}\left[\mathrm{Ca}^{2+}\right]_{\mathrm{e}}$ to $1.76 \pm 0.12 \Delta F / F_{0}$ in $4 \mathrm{mM}\left[\mathrm{Ca}^{2+}\right]_{\mathrm{e}}$ (paired t test, $p<$ $0.0001, n=27$ boutons in 23 slices) indicating higher glutamate concentrations in the synaptic cleft under conditions of higher release probability. Values are given as mean \pm SEM. c) The probability of successes was correlated with the amplitude of success trials in a non-linear fashion. d) In $2 \mathrm{mM}\left[\mathrm{Ca}^{2+}\right]_{\mathrm{e}}$, the synaptic release probability of individual synapses ranged from 0.17 to 0.99 . The amplitude of success trials was similar for boutons with low synaptic release probability but rose steeply for $p_{\text {syn }}>0.8$.

\section{Mapping the spatial location of individual fusion events}

137 Large iGluSnFR signals from high $p_{\text {syn }}$ boutons suggest multivesicular release, but are all

138 vesicles released from a single active zone? To map the spatial location of individual release

139 events (fusion site), we acquired rapid frame scans $(16 \times 16$ pixels, $62.5 \mathrm{~Hz})$ at high

140 magnification (Fig. 3a). The red (cytoplasmic) fluorescence was used to align all frames post

141 hoc. Putative vesicle fusion events were localized by fitting a two-dimensional Gaussian kernel

142 to the green fluorescence in all success trials (Fig. 3b). We classified trials as successes or

143 failures based on the standard deviation of fluorescence before stimulation ( $2 \sigma$ criterion). In 
144 success trials, the putative fusion events were typically localized to a small region (Fig. $3 \mathrm{c}, 1 \mathrm{mM}$ $145\left[\mathrm{Ca}^{2+}\right]_{\mathrm{e}}$ ). Under conditions of increased release probability (Fig. 3c, $4 \mathrm{mM}\left[\mathrm{Ca}^{2+}\right]_{\mathrm{e}}$ ), fusion events 146 remained localized to the same sub-region of the bouton. The same fitting procedure applied to 147 failure trials resulted in a randomly distributed 'noise' map (Fig. 3c, failures), suggesting that the $1482 \sigma$ criterion correctly distinguished failures from successes. A similar random distribution was 149 seen when the frame before stimulation was analyzed (Fig. 3c, baseline). The spatial distribution 150 of fusion events was often elliptical, suggesting a synaptic contact on the side of the bouton (Fig. $1513 d$ ). We fitted the distribution of the localized fusion events with an ellipse including $95 \%$ of the 152 successes. We assume that the long axis of the fusion site distribution (range: 0.29 to $0.98 \mu \mathrm{m}$ )

153 represents the true diameter of the active zone whereas the short axis is the result of its

154 geometrical projection. To calibrate our localization method, we acquired series of images from a 155 green fluorescent microsphere next to a bouton, which resulted in more confined and circular 156 maps (Fig. 3e). From these bead measurements, which were matched to the photon count of 157 iGluSnFR signals, we estimate our localization precision to be $0.05 \pm 0.01 \mu \mathrm{m}$, which is better 158 than the lateral resolution of our microscope $(0.48 \pm 0.02 \mu \mathrm{m})$. In high $\left[\mathrm{Ca}^{2+}\right]_{\mathrm{e}}$, the same boutons 159 displayed significantly more confined event distributions (Fig. 3f). In high $\left[\mathrm{Ca}^{2+}\right]_{\mathrm{e}}$, simultaneous 160 fusion of multiple vesicles occurs frequently. Under these conditions, the glutamate cloud is not 161 generated by a single 'point source', and we map the centroid of multiple fusion events. The 162 distribution of centroids is expected to be less variable than the distribution of individual fusion 163 events, explaining the tighter spatial distribution in high $\left[\mathrm{Ca}^{2+}\right]_{\mathrm{e}}$. Most importantly, the mapping 164 experiments confirmed that at the majority of boutons, iGluSnFR signals were generated by a 165 single active zone (Extended Data Fig. 3). In a few experiments, we found more than one active 166 site on a single bouton (Extended Data Fig. 4). Such multi-synapse boutons, which could also be 167 identified in line scan mode, were excluded from further analysis. Taken together, our 168 measurements show that synaptic vesicles are released at variable positions within a confined 169 area (single active zone). Whether vesicle release sites were further organized into discrete 170 nanodomains within the active zone, as has been shown for synapses grown on glass cover 171 slips $^{6}$, could not be determined due to the intrinsic mechanical instability of brain tissue. 
a

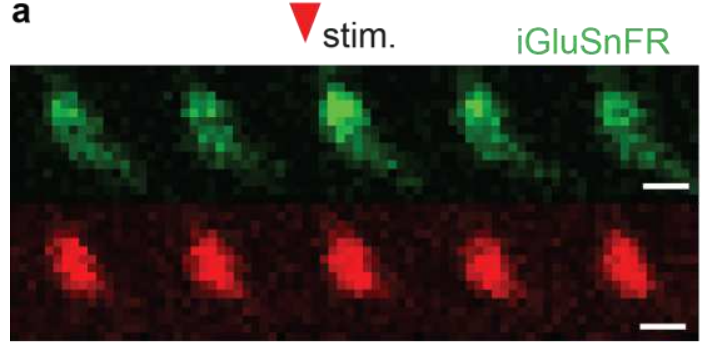

tdimer-2

b

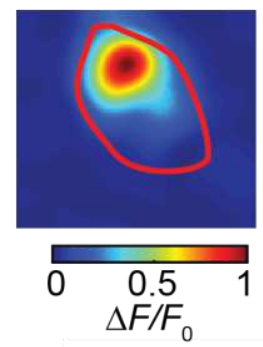

d

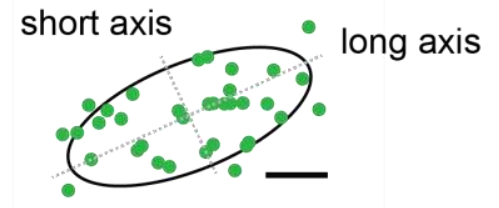

e
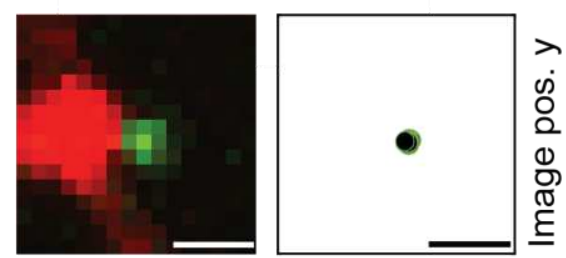

Image pos. $\mathrm{x}$ c

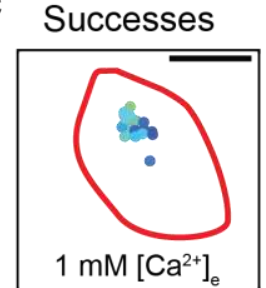

Failures

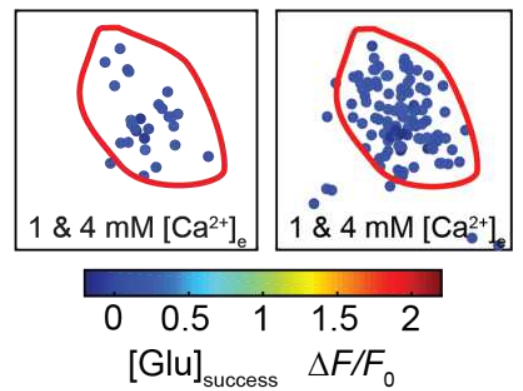

f

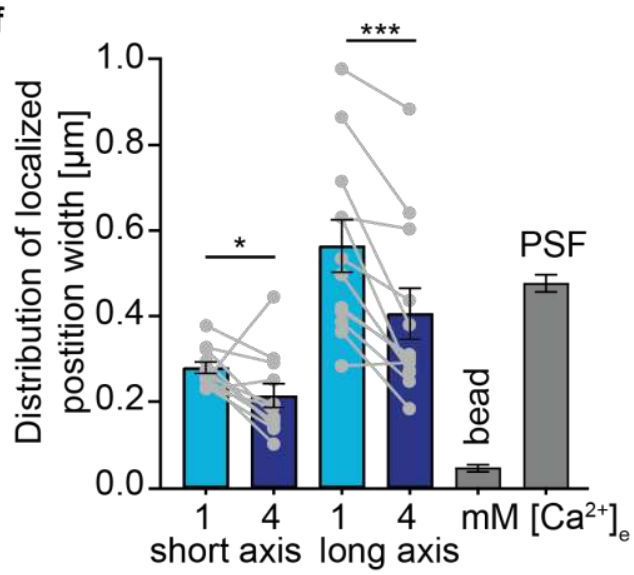

Figure 3: Localizing the fusion site. a) Time-lapse (frame rate $62.5 \mathrm{~Hz}$ ) of a bouton releasing glutamate in response to an individual AP. Scale bar, $1 \mu \mathrm{m}$. b) (Left) top view of average responses of signals classified as successes. (Right) side view of the resulting 2D-Gaussian kernel fits. c) (Upper left) center positions of 2D-Gaussian kernel fits in success trials in ACSF containing $1 \mathrm{mM}\left[\mathrm{Ca}^{2+}\right.$ e. (Upper right) center positions of 2D-Gaussian kernel fit in success trials in ACSF containing $4 \mathrm{mM}\left[\mathrm{Ca}^{2+}\right]$ e. (Lower left) center positions of 2D-Gaussian kernel fits in failure trials (below $2 \sigma$ of the baseline). (Lower right) center positions of 2D-Gaussian kernel fits in failure frames before the stimulation in ACSF containing $1 \mathrm{mM}\left[\mathrm{Ca}^{2+}\right]_{\mathrm{e}}$ and ACSF containing $4 \mathrm{mM}\left[\mathrm{Ca}^{2+}\right]_{\mathrm{e}}$. Scale bar, $1 \mu \mathrm{m}$. d) Example of a fitted ellipse including $95 \%$ of localized successes (green dots) from a single bouton. The length of the short and long axis of the fitted ellipse of successes measured in $1 \mathrm{mM}\left[\mathrm{Ca}^{2+}\right]_{\mathrm{e}}$ and $4 \mathrm{mM}\left[\mathrm{Ca}^{2+}\right]_{\mathrm{e}}$ are used to test for a relative enlarging of the release area in $4 \mathrm{mM}\left[\mathrm{Ca}^{2+}\right]_{\mathrm{e}}$ indicative of a multisynapse bouton. Scale bar, $128 \mathrm{~nm}$. e) (Left) single plane of a twophoton microscope image of a bouton (tdimer2) next to a yellow-green fluorescent microspheric bead $(0.17 \mu \mathrm{m})$. (Right) center positions of 2D-Gaussian kernel fits to the fluorescent signal of a bead. 50 consecutive images were acquired at $62.5 \mathrm{~Hz}$. Scale bars, $1 \mu \mathrm{m}$. f) Histogram of short and long axis of the fitted ellipse to the localized responses and FWHM distribution of the localized bead and PSF measurement. Success localization short axis, 0.28 $\pm 0.01 \mu \mathrm{m}$ in $1 \mathrm{mM}\left[\mathrm{Ca}^{2+}\right]_{\mathrm{e}}$ and $0.21 \pm 0.03 \mu \mathrm{m}$ in $4 \mathrm{mM}\left[\mathrm{Ca}^{2+}\right]_{\mathrm{e}}$ (Wilcoxon test, $p=0.04, n=12$ boutons); success localization long axis, $0.56 \pm 0.06 \mu \mathrm{m}$ in $1 \mathrm{mM}\left[\mathrm{Ca}^{2+}\right]_{\mathrm{e}}$ and $0.41 \pm 0.06 \mu \mathrm{m}$ in $4 \mathrm{mM}\left[\mathrm{Ca}^{2+}\right]_{\mathrm{e}}$ (Wilcoxon test, $p=0.001, n$ = 12 boutons); bead localization $0.05 \pm 0.01 \mu \mathrm{m}$; PSF bead, $0.48 \pm 0.02 \mu \mathrm{m})$. Values are given as mean \pm SEM. 


\section{Desynchronized release events reveal quantal size}

193 Although previous studies using postsynaptic measurements of AMPAR currents found evidence

194 for multivesicular release at Schaffer collateral synapses ${ }^{14,20}$, it has not been possible to

195 compare the amplitude of evoked responses to the amplitude of spontaneous fusion events

196 ('minis') at the same synapse. To perform a classical quantal analysis, the size of the quantum

197 (q) has to be known. We therefore replaced extracellular $\mathrm{Ca}^{2+}$ with $\mathrm{Sr}^{2+}$ to desynchronize vesicle

198 fusion events ${ }^{21}$ while monitoring glutamate transients at single boutons (Fig. 4). $\mathrm{Sr}^{2+}$ is known to

199 lead to asynchronous release due to its low affinity for synaptotagmin- $1^{22}$ and its slow clearance

200 from the presynaptic terminal ${ }^{23}$. As expected, large-amplitude glutamate release events occurred 201 with high probability in $4 \mathrm{mM}\left[\mathrm{Ca}^{2+}\right]_{\mathrm{e}}$. When artificial cerebrospinal fluid (ACSF) containing $4 \mathrm{mM}$

$202 \mathrm{Ca}^{2+}$ was slowly replaced by ACSF containing $4 \mathrm{mM} \mathrm{Sr}^{2+}$, evoked glutamate transients started to 203 disintegrate into smaller events of relatively uniform amplitude. When $4 \mathrm{mM} \mathrm{Sr}^{2+}$ was fully

204 washed-in, evoked responses completely disappeared while baseline fluorescence became very

205 noisy. The amplitude histogram shows clear separation between evoked responses (Fig. 4b and

206 c, blue bars) and delayed events (green bars). We interpret this sequence of events during

207 wash-in as evoked multivesicular release, delayed release of individual vesicles under the

208 influence of $\left[\mathrm{Sr}^{2+}\right]_{\mathrm{e}}$, and finally, incomplete fusion in the absence of $\left[\mathrm{Ca}^{2+}\right]_{\mathrm{e}}$. The quantal

209 amplitude determined with this method was $q=96 \% \pm 15 \% \Delta F / F_{0}$ ( $n=3$ boutons).

210

211 The dynamic range of postsynaptic responses

212 Our optical measurements demonstrate a steep dependence of release probability on [Ca $\left.{ }^{2+}\right]_{\mathrm{e}}$.

213 Would AMPA receptors report increased glutamate concentrations as larger currents? To

214 measure the strength of unitary connections, we performed dual patch-clamp recordings from

215 connected pairs of CA3 and CA1 pyramidal cells under NMDAR block (10 $\mu$ M CPP-ene) (Fig

$2165 a)$. For each pair, we recorded EPSCs in $1 \mathrm{mM}$ and in $4 \mathrm{mM}\left[\mathrm{Ca}^{2+}\right]_{\mathrm{e}}$, switching the sequence

217 (low to high / high to low $\left[\mathrm{Ca}^{2+}\right]_{\mathrm{e}}$ ) between experiments. The boosting of AMPA EPSCs by high

$218\left[\mathrm{Ca}^{2+}\right]_{\mathrm{e}}$ was similar to the boosting of iGluSnFR signals (Fig $5 \mathrm{c}$ ), suggesting that AMPARs were

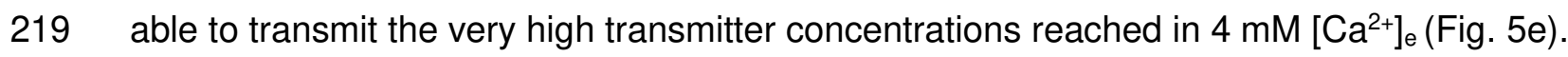



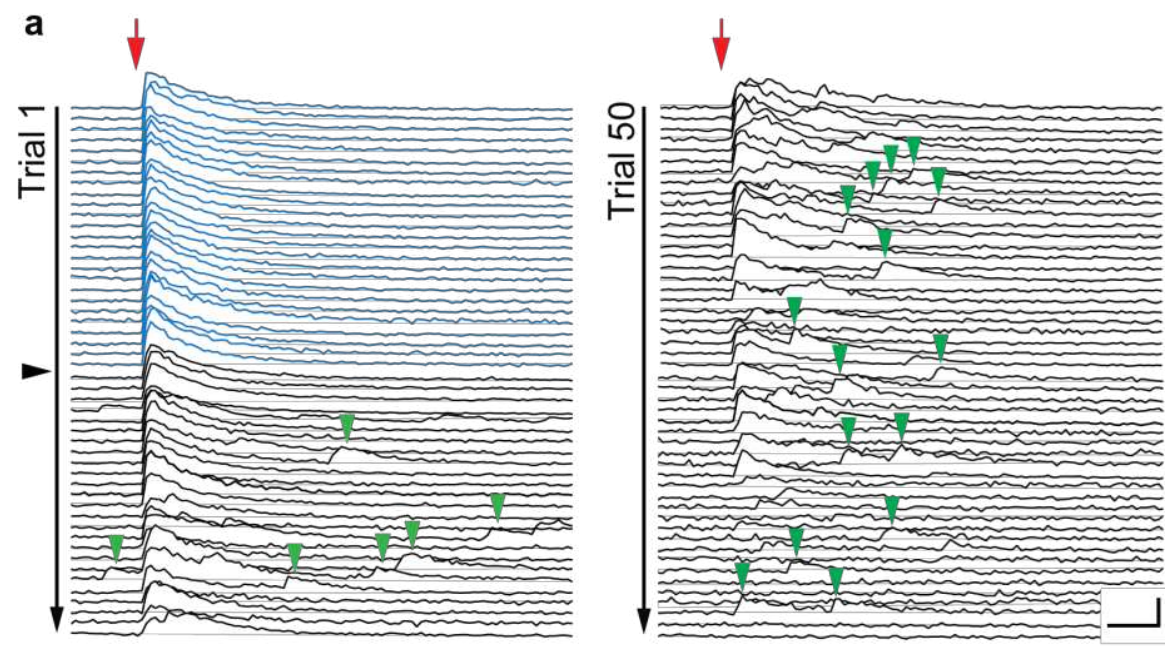

b

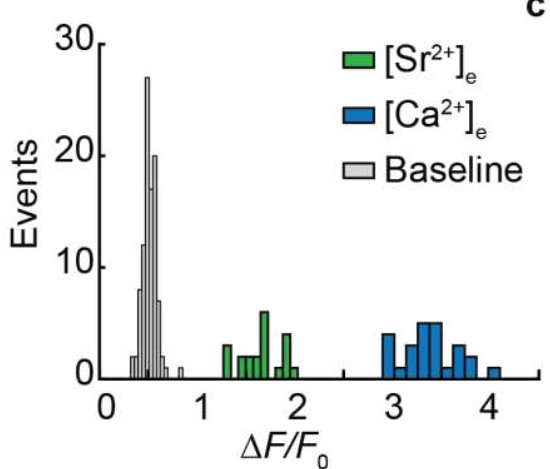

C

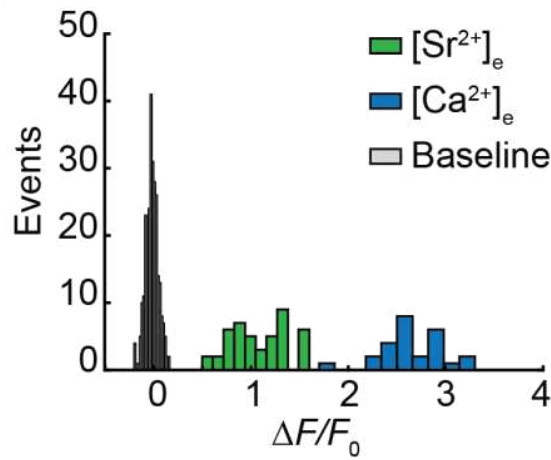

Figure 4: Desynchronized release events reveal quantal size. a) iGluSnFR changes in fluorescence (single bouton) in response to single APs in ACSF containing $4 \mathrm{mM}\left[\mathrm{Ca}^{2+}\right]_{\mathrm{e}}$ (blue traces). Red arrow indicates stimulation onset. Synaptic release probability was 1 (no failures). The black arrowhead indicates the start of $\left.4 \mathrm{mM}^{\left[\mathrm{Sr}^{2+}\right.}\right] \mathrm{e}$. wash-in. During wash-in of $4 \mathrm{mM}\left[\mathrm{Sr}^{2+}\right]_{\mathrm{e}}$ discrete events appear in the wake of the stimulation (green arrowheads). Scale bar, 2 $\Delta F / F_{0}$ and $20 \mathrm{~ms}$. b) Amplitude histogram of evoked responses measured in $4 \mathrm{mM}\left[\mathrm{Ca}^{2+}\right]_{\mathrm{e}}$ (blue bars) and delayed

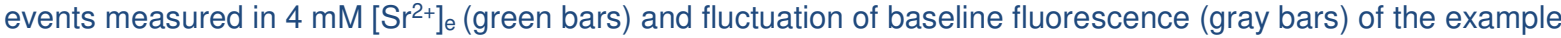

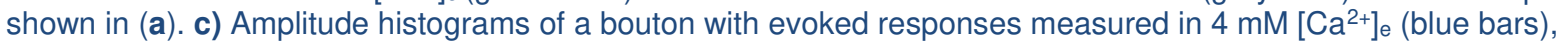

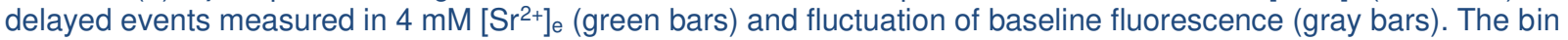
size is smaller for the baseline fluctuations for display purposes.

Reducing AMPAR occupancy by the competitive antagonist $\gamma$-DGG $(10 \mathrm{mM})$ did not increase the difference between low and high $\left[\mathrm{Ca}^{2+}\right]_{\mathrm{e}}$ EPSCs, suggesting that AMPARs were not saturated by stimulation in $4 \mathrm{mM}\left[\mathrm{Ca}^{2+}\right]_{e}$ (Extended Data Fig. 5). We verified that blocking NMDARs did not affect the release of glutamate (Extended Data Fig. 6). Furthermore, in paired recordings, expression of iGluSnFR or a membrane-bound GFP in the postsynaptic cell did not change synaptic strength or paired-pulse ratio (Extended Data Fig. 7). 
a AMPARs

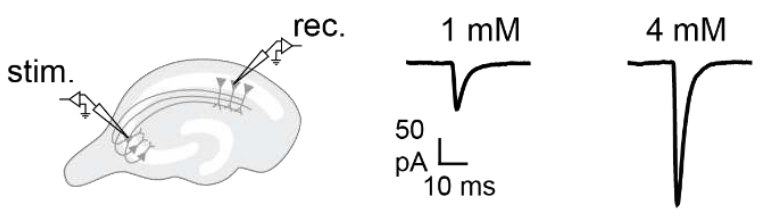

C

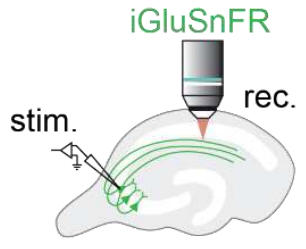

b

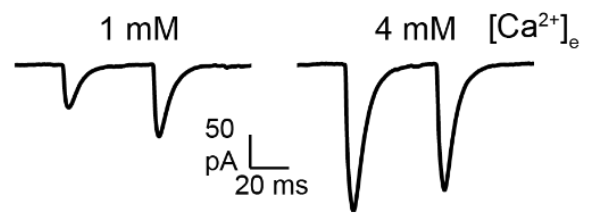

d
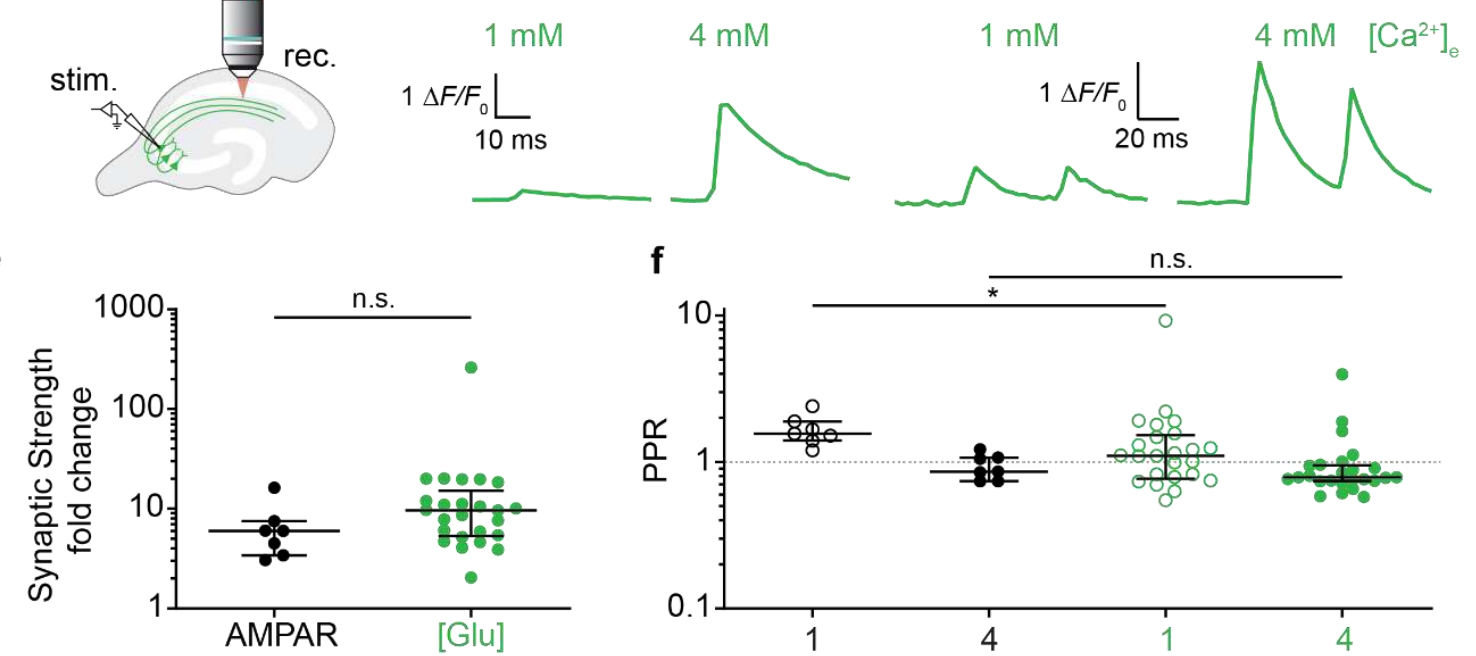

$\mathbf{f}$

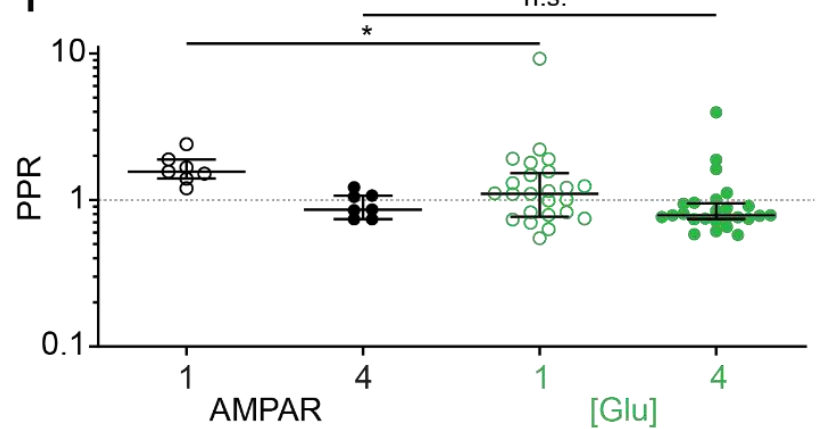

Figure 5: Multivesicular release increases the gain and the signal to noise ratio of synapses. a) EPSCs were measured by dual patch-clamp recordings from connected CA3-CA1 pyramidal cell pairs. Perfusion was switched from $1 \mathrm{mM}$ to $4 \mathrm{mM}\left[\mathrm{Ca}^{2+}\right]_{\text {e }}$, leading to increased EPSC amplitudes. Traces show EPSCs (average of $\sim 40$ trials) from the CA1 pyramidal cell. b) Representative EPSCs (average of $\sim 40$ trials) in response to the presynaptic paired-pulse stimulation (ISI $48 \mathrm{~ms}$ ) recorded in $1 \mathrm{mM}$ and $4 \mathrm{mM}\left[\mathrm{Ca}^{2+}\right]$ e. c) iGluSnFR signals were measured as described previously (Fig. 1). Traces show the evoked change in fluorescence (average of $\sim 60$ trials) from a single bouton in CA1 stratum radiatum. The switching sequence (low - high $\left[\mathrm{Ca}^{2+}\right]_{\mathrm{e}} /$ high - low $\left[\mathrm{Ca}^{2+}\right]_{\mathrm{e}}$ ) was reversed between experiments. d) Representative iGluSnFR signals (average of $\sim 60$ trials) from a single bouton in CA1 stratum radiatum in response to the paired-pulse stimulation. e) Increasing $\left[\mathrm{Ca}^{2+}\right]_{\mathrm{e}}$ from 1 to $4 \mathrm{mM}$ increased the median amplitude of AMPAR-mediated EPSCs by a factor of 6.02, interquartile range (IQR): 3.4-7.5 fold, $n=7$ pairs, the median iGluSnFR response by 9.63 fold, IQR: $5.3-15.2$ fold, $n=25$ boutons. There is no significant difference between the fold change in AMPARs and iGluSnFR responses (Mann-Whitney, $p=0.0832$ ). f) EPSCs showed pairedpulse facilitation in $1 \mathrm{mM}\left[\mathrm{Ca}^{2+}\right]_{\mathrm{e}}$, (median PPR: 156\%, IQR: 1.4\%-1.9\%, $n=7$ pairs) and paired-pulse depression in 4 $\mathrm{mM}\left[\mathrm{Ca}^{2+}\right]_{\mathrm{e}}$ (median PPR: 86\%, IQR: 74\%-107\%, $n=7$ pairs). iGluSnFR responses showed paired-pulse facilitation in $1 \mathrm{mM}\left[\mathrm{Ca}^{2+}\right]_{\mathrm{e}}$ (median PPR: 110\%, IQR: 77\%-152\%, $n=25$ boutons) and paired-pulse depression in $\left.4 \mathrm{mM}^{\mathrm{C}} \mathrm{Ca}^{2+}\right]_{\mathrm{e}}$ (median PPR: 79\%, IQR: 74\%-95\%, $n=25$ boutons). There is a significant difference between the AMPARs PPR and

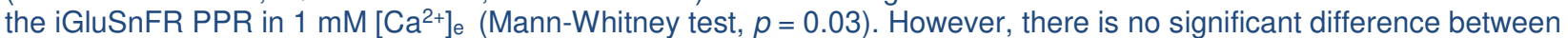
the AMPARs PPR and the iGluSnFR PPR in $4 \mathrm{mM}\left[\mathrm{Ca}^{2+}\right]$ e (Mann-Whitney test, $p=0.37$ ). Value with a PPR $=0$ is not plotted for display purpose but was used for statistics. Values are plotted as median with IQR.

Due to vesicle pool depletion and AMPAR desensitization, we expected to see some degree of depression at higher stimulation frequencies, especially under conditions of high release probability (4 $\left.\mathrm{mM}\left[\mathrm{Ca}^{2+}\right]_{\mathrm{e}}\right)$. We therefore compared the paired-pulse ratios from patch-clamp recordings of connected CA3-CA1 pairs and iGluSnFR signals from individual boutons. In $1 \mathrm{mM}$ $\left[\mathrm{Ca}^{2+}\right]_{e}$, EPSCs showed paired-pulse facilitation (PPR $=156 \%$ ), which was absent in $4 \mathrm{mM}$ $\left[\mathrm{Ca}^{2+}\right]_{\mathrm{e}}(\mathrm{PPR}=86 \%$, Fig. 5b and $\mathrm{f})$. iGluSnFR responses showed weak facilitation in $1 \mathrm{mM}$ $\left[\mathrm{Ca}^{2+}\right]_{\mathrm{e}}(\mathrm{PPR}=110 \%)$ and depression in $4 \mathrm{mM}\left[\mathrm{Ca}^{2+}\right]_{\mathrm{e}}(\mathrm{PPR}=79 \%$, Fig. 5d and f), consistent 
with the expected partial depletion of readily-releasable vesicles under high release probability

264 conditions. We conclude that Schaffer collateral synapses are able to maintain a fairly linear

265 paired pulse response over a $\sim 10$-fold change in synpatic strength, which is remarkable.

267 Non-linear response of iGluSnFR to glutamate release

268 Fusion of a single vesicle produces an extremely localized glutamate release into the synaptic 269 cleft that rapidly disperses through diffusion. To explore how diffusing glutamate molecules, 270 interact with iGluSnFR and postsynaptic AMPARs, we implemented a Monte Carlo simulation 271 consisting of a glutamatergic bouton contacting a dendritic spine surrounded by astrocytes.

272 Simulated fusion of a transmitter vesicle in the center of the synaptic cleft produced a local cloud 273 of glutamate that filled the entire cleft within $10 \mu \mathrm{s}$ and was largely cleared $100 \mu$ s later (Fig. 6a).

274 Consequently, iGluSnFR molecules became bound (and fluorescent) and doubly bound

275 AMPARs opened (Fig. 6b). The model allowed us to explore how different orientations of the 276 synapse with respect to the optical axis would affect the amplitude of iGluSnFR signals (Fig. 6c

277 and d). The largest signal in response to fusion of a single vesicle (quantal amplitude, $q$ ) is 278 generated when both spine and axon are in the focal plane, aligning the synaptic cleft with the 279 optical axis. A synapse where spine or axon are tilted with respect to the focal plane will produce 280 smaller signals, since more extrasynaptic iGluSnFR molecules are inside the PSF and thus elevate the baseline fluorescence $\left(F_{0}\right)$, decreasing the relative change in fluorescence $\left(\Delta F / F_{0}\right)$. This was an important insight, considering that a horizontal orientation of axons and spines is typical for organotypic slice cultures ${ }^{24}$. Nevertheless, we still expect variability in $q$ between individual boutons due to variable spine orientations. Thus, when modeling iGluSnFR amplitude distributions, we treated $q$ as a free parameter (search range: $50-200 \% \Delta F / F_{0}$ ). The fraction of bright iGluSnFR and fraction of open AMPARs was low after release of a single vesicle (Fig. 6e), assuming 3000 molecules of glutamate per vesicle ${ }^{25}$. Simulated release of multiple vesicles increased the bound fraction of iGluSnFR and AMPARs. The saturation process was well approximated by hyperbolic functions

Equation 1: $r=\frac{[\mathrm{Glu}]}{K_{\mathrm{d}}+[\mathrm{Glu}]}$

291 where $r$ corresponds to the fraction of iGluSnFR molecules (or AMPARs) bound to glutamate.

292 Fraction of bound iGluSnFR and open AMPARs upon release of a single SV were $20 \%$ and $29317 \%$, respectively. 
a

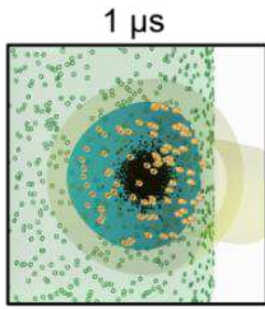

$100 \mu \mathrm{s}$

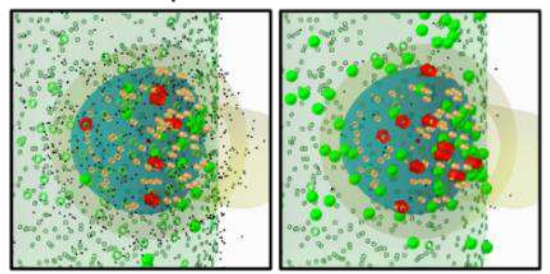

- glutamate oiGluSnFR

- iGluSnFR-glu dim iGluSnFR-glu $_{\text {bright }}$

AMPAR oopen AMPARs

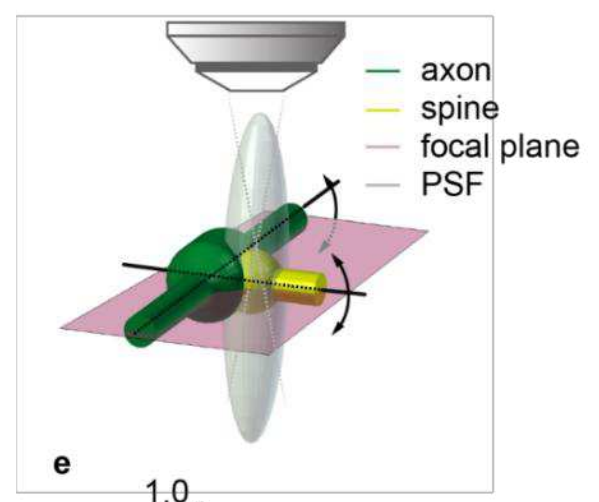

e

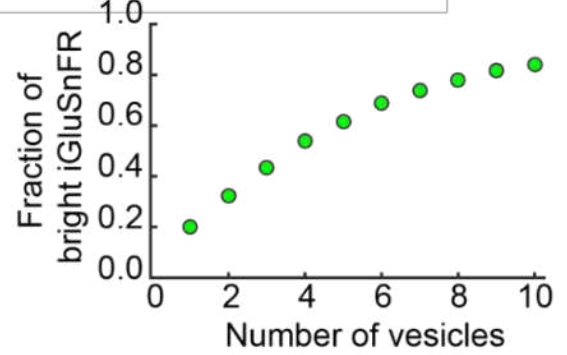

b

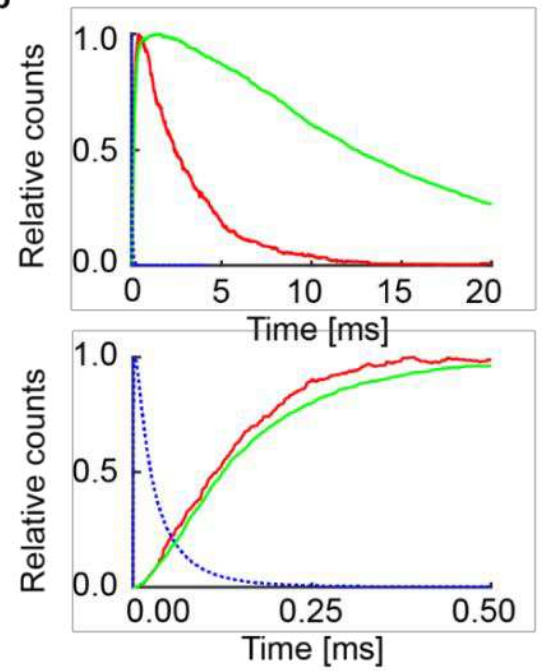

..... glutamate - iGluSnFR-glu ${ }_{\text {bright }}$

- open AMPARs
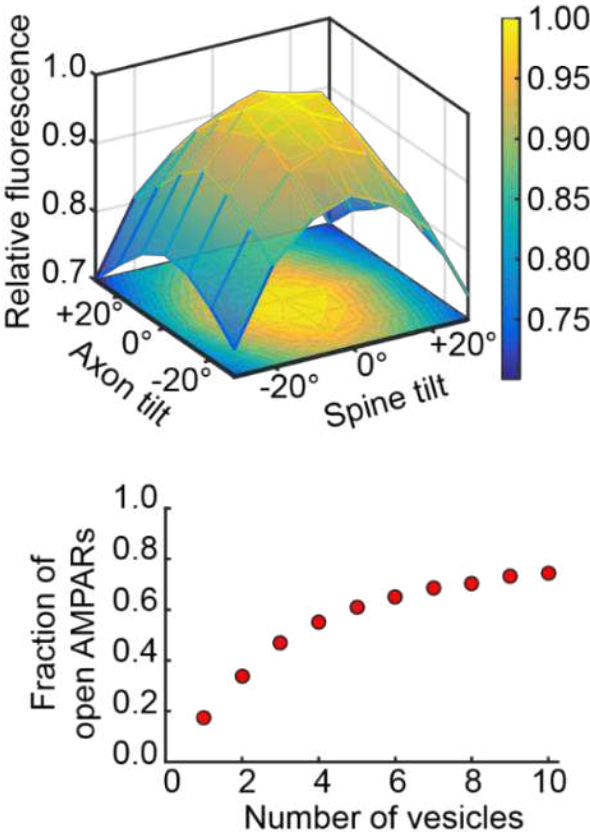

Figure 6: Monte-Carlo-simulation of glutamate diffusion in the synaptic cleft. a) Simulated glutamate dynamics within the synaptic cleft upon vesicle release. The model consists of a presynaptic terminal with iGluSnFR molecules opposed to a spine with AMPARs randomly distributed in a disc of $300 \mathrm{~nm}$, separated by a $20 \mathrm{~nm}$ synaptic cleft. The synapse is embedded in a network of astrocytes equipped with glutamate transporters. b) Simulated reaction time profile of AMPARs opening and iGluSnFR in a bright fluorescent state upon release of a single vesicle containing 3000 molecules of glutamate (upper panel) and close-up view of the kinetics from 0 to 50 ms (lower panel). c) Simulated fluorescence measurements under different orientations of axon and spine with respect to the optical axis. iGluSnFR fluorescence was evaluated inside the PSF. Tilting the axon or the spine reduces $\Delta F / F_{0}$, as more iGluSnFR molecules outside the synaptic cleft fall within the PSF, contributing to the resting fluorescence $F_{0}$. d) Normalized fluorescence transient with respect to tilted positions of axon or spine. e) Fraction of bright iGluSnFR bound to glutamate (left panel) and fraction of open AMPARs (right panel) after release of 1-10 vesicles (3000 glutamate molecules per vesicle). Occupancy of iGluSnFR and AMPA receptors is similar after a single vesicle release, resulting in similar saturation curves. 
310 The histograms of iGluSnFR responses from individual boutons often showed multiple peaks.

311 Distinct quantal peaks have been observed in EPSC distributions from large mossy fiber

312 synapses on CA3 pyramidal cells ${ }^{26}$, but are much less clear at CA3-CA1 connections ${ }^{27}$. If these

313 peaks indicate the simultaneous release of two or more vesicles in response to a single

314 presynaptic AP, there are clear predictions about the amplitude and spacing of the peaks: The

315 amplitude of the peaks would be expected to follow binomial statistics, as famously shown for

316 endplate potentials ${ }^{28}$. Due to the saturation of iGluSnFR at $440 \% \Delta F / F_{0}$, however, quantal peaks

317 should not be equidistant, but compressed according to a hyperbolic saturation function

318 (Equation 1).

319 To investigate release statistics in more detail, we performed optical quantal analysis from the

320 iGluSnFR signals of individual Schaffer collateral boutons monitored 1 and $4 \mathrm{mM}\left[\mathrm{Ca}^{2+}\right]_{\mathrm{e}}$. The

321 fluorescence trace in every trial was fit with a kernel (exponential decay function) to extract the

322 peak amplitude. The resulting histograms were quite variable, sometimes displaying multiple

323 peaks. To extract the three quantal parameters $N, p_{\text {ves }}$, and $q$ from the response histograms, we

324 generated predictions (probability density functions) for all possible parameter triplets

325 (exhaustive search) to find the combination of parameters that explains best the histogram of

326 iGluSnFR signals. First, for every combination of $N$ and $p_{\text {ves, }}$, we calculated the binomial

327 probabilities for the different outcomes (failures, univesicular and various multivesicular events,

328 Fig. 7a). From the baseline fluorescence distribution of the synapse in question, we extracted

329 the expected variability of failure fluorescence (width of the Gaussian). As photon shot noise

330 increases with the square root of the number of detected photons, we added appropriate

331 amounts of 'noise' to the expected quantal peaks leading to a broadening of the individual

332 Gaussians as the signal increased. To account for partial saturation of iGluSnFR at high

333 glutamate concentrations $\left(\left(F_{\max }-F_{0}\right) / F_{0}=440 \%\right.$, see methods), we spaced the expected

334 quantal peaks not as integer multiples of $q$, but according to the saturation function (Equation 1).

335 For every prediction, we scaled the amplitude to match the number of observations (histogram)

336 and calculated the mean square error. We observed that different combinations of $N$ and $p_{\text {ves }}$

337 generated near-identical fits, as there was no 'cost' associated with increasing $N$ in the model.

338 We decided to select the prediction with the smallest number of vesicles that was within $2 \%$ of

339 the minimum mean square error as the most parsimonious biophysical mechanism for the

340 synapse in question. 
a
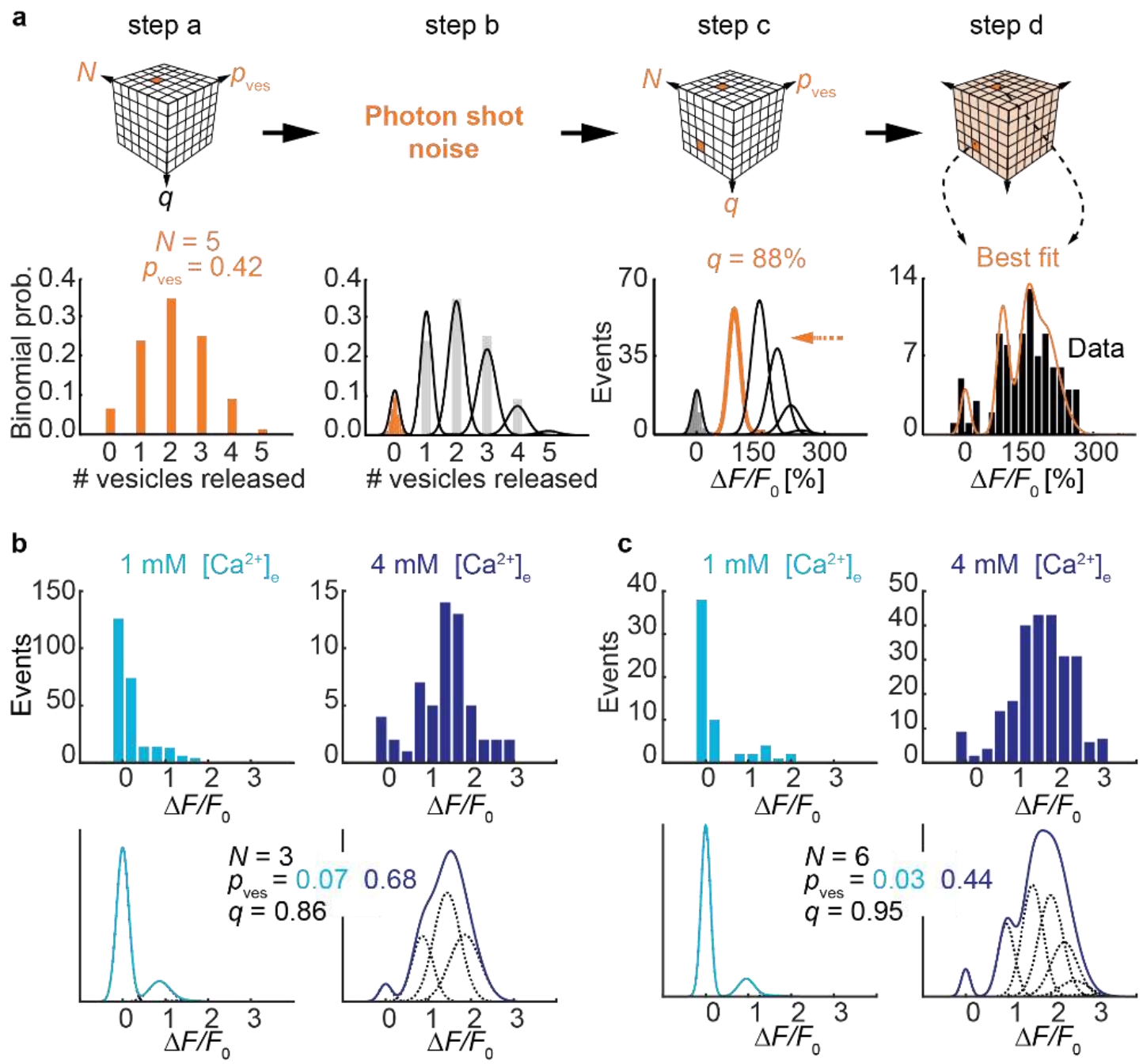

d
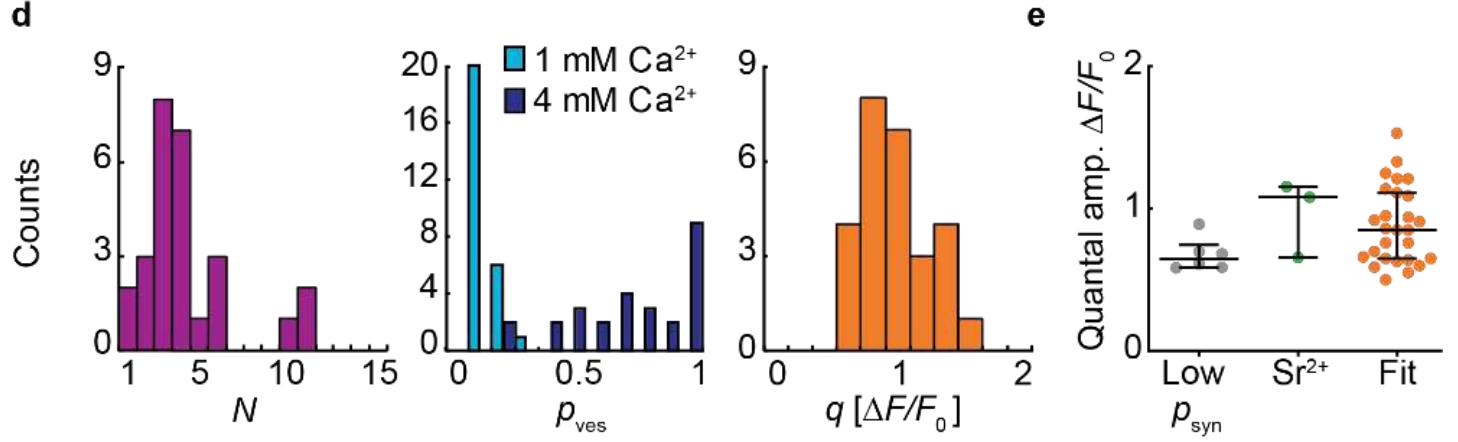

Figure 7: Extracting $N, p_{\text {ves }}$ and $q$ of Schaffer collateral synapses using a binomial model. a) Schematic view of the exhaustive search of the quantal parameters $N$, p pes and $q$. For a combination of $N$ and pres, the binomial probabilities of the possible outcomes were calculated (here: $N=5$ vesicles and $p_{\text {ves }}=0.42$ ). Without stimulation, amplitudes were normally distributed around zero (orange bars). The width of this noise distribution, a function of the number of photons detected from a particular bouton, was used to determine the width of the expected Gaussian probability density functions. A chosen quantal amplitude $q$ (here: $88 \% \Delta F / F_{0}$ ) sets the spacing of the Gaussian probability density functions. iGluSnFR saturation (max. $\Delta F / F_{0}=440 \%$ ) was considered when setting the expected amplitudes of multi-quantal events, leading to a compression (orange arrow) of the individual Gaussians. The resulting probability density function (sum of the Gaussians) was compared to the measured amplitude distribution of a single bouton (black bars, recorded in $2 \mathrm{mM}\left[\mathrm{Ca}^{2+}\right]_{\mathrm{e}}$ ). The root mean square (RMS) error was calculated and the best fit (shown here) was selected to determine the synaptic parameters. 
b-c) Single bouton response distributions recorded in $1 \mathrm{mM}\left[\mathrm{Ca}^{2+}\right]_{\mathrm{e}}$ and $4 \mathrm{mM}\left[\mathrm{Ca}^{2+}\right]_{\mathrm{e}}$. Two examples from two different slice cultures. The binomial fitting procedure was applied to both histograms, searching for the best combined fit under the condition that $N$ and $q$ had to be identical in $1 \mathrm{mM}\left[\mathrm{Ca}^{2+}\right]_{\mathrm{e}}$ and $4 \mathrm{mM}\left[\mathrm{Ca}^{2+}\right]_{\mathrm{e}}$ while $p_{\text {ves }}$ could be different. Best fit probability density functions and extracted parameters are shown below the experimental data. d) Extracted quantal parameters, $N$ median: 4 vesicles, IQR: $3-5$ vesicles; pres in $1 \mathrm{mM}\left[\mathrm{Ca}^{2+}\right]_{\mathrm{e}}$ median: 0.07, IQR: 0.03-0.11; $p_{\text {ves }}$ in $4 \mathrm{mM}\left[\mathrm{Ca}^{2+}\right]_{\mathrm{e}}$ median: 0.71, IQR = 0.45-0.96; $q$ median $=0.85 \Delta F / F_{0}, \mathrm{IQR}: 0.65-1.11 \Delta F / F_{0}, n=27$ boutons. The numbers delimit the right edge of their respective bin. e) Estimating quantal amplitude by three different approaches using independent datasets. Measurements report no significant difference (Kruskal-Wallis test, $p=0.13$ ) between the quantal sizes. $p_{\text {syn }}<0.5$ boutons in $2 \mathrm{mM}\left[\mathrm{Ca}^{2+}\right]$ e median: $0.65 \Delta F / F_{0}$, IQR: 0.59-0.75 $\Delta F / F_{0}, n=6$ boutons; delayed events in $4 \mathrm{mM}\left[\mathrm{Sr}^{2+}\right]_{\mathrm{e}}$ wash-in experiments median: $1.08 \Delta F / F_{0}$, IQR: 0.66-1.15 $\Delta F / F_{0}, n=3$ boutons; binomial fitting procedure median: $0.85 \Delta F / F_{0}$, IQR: $0.65-1.11 \Delta F / F_{0}, n=27$ boutons. Values are given as median with IQR.

342 To further constrain the fitting procedure, the algorithm had to find values for $q$ and for $N$ that 343 could account for the histogram of responses in $1 \mathrm{mM}\left[\mathrm{Ca}^{2+}\right]_{\mathrm{e}}$ and for the histogram count 344 measured in $4 \mathrm{mM}\left[\mathrm{Ca}^{2+}\right]_{e}$. The quantal size and number of release-ready vesicles are not 345 expected to change with $\left[\mathrm{Ca}^{2+}\right]_{\mathrm{e}}$. Only $p_{\text {ves }}$ was allowed to vary between the low and high $\left[\mathrm{Ca}^{2+}\right]_{\mathrm{e}}$ 346 condition. The fitting results provided a convincing explanation why some boutons showed 347 multiple peaks in $4 \mathrm{mM}\left[\mathrm{Ca}^{2+}\right]_{\mathrm{e}}$ while those multiple peaks were not apparent in $1 \mathrm{mM}\left[\mathrm{Ca}^{2+}\right]_{\mathrm{e}}$

348 (Fig. 7b and c, upper panels). Due to partial saturation of iGluSnFR at high glutamate

349 concentration, quantal peaks for three or more simultaneously released vesicles are not 350 resolved, but compressed into a broad peak (Fig. $7 \mathrm{~b}$ and c, lower panels). In our sample of 27

351 boutons, the estimated number of docked vesicles ranged from 1 to 11 , with only 2 boutons 352 having just one docked vesicle. Changing $\left[\mathrm{Ca}^{2+}\right]_{\mathrm{e}}$ from 1 to $4 \mathrm{mM}$ increased $p_{\mathrm{ves}} 9.9$-fold (median 353 change). A typical bouton in $1 \mathrm{mM}\left[\mathrm{Ca}^{2+}\right]_{\mathrm{e}}$ released a single vesicle in $20 \%$ of trials and multiple 354 vesicles in only $\sim 4 \%$ of trials. In $4 \mathrm{mM}\left[\mathrm{Ca}^{2+}\right]_{e}$, multivesicular events were much more common $355(77 \%)$ and failures were rare (11\%).

356 We estimated the iGluSnFR response to the release of a single vesicle $(q)$ with three different 357 approaches of increasing complexity: 1) by analyzing the amplitude of successes under low 358 release probability conditions, 2) by measuring the amplitude of desynchronized events during $359\left[\mathrm{Sr}^{2+}\right]_{\mathrm{e}}$ replacement experiments, and 3) by fitting a binomial model to the complete distribution of 360 successes and failures from a single bouton (Fig. 7e). The resulting estimates of $q$ were very 361 consistent between methods and between individual experiments, indicating that the presynaptic 362 quantum is indeed of constant size at glutamatergic synapses (although postsynaptic responses 363 may vary). It is important to note that the absolute amplitude $\left(\Delta F / F_{0}\right)$ of $q$ depends not only on 364 the indicator, but also on the spatial and temporal resolution of the microscope as it is trying to 365 catch the peak fluorescence caused by a rapidly diffusing cloud of glutamate. 
367 Lastly, we used our dataset to determine which synaptic parameter has the strongest impact on 368 (pre-)synaptic strength. For each synapse, we calculated its synaptic strength as the product of 369 quantal parameters $\left(p_{\text {ves }} \times N \times q\right)$ under low and under high $\left[\mathrm{Ca}^{2+}\right]_{\mathrm{e}}$ conditions. Compared to 370 simply averaging all iGluSnFR responses, this removes the compressive effect of indicator 371 saturation. In $1 \mathrm{mM}\left[\mathrm{Ca}^{2+}\right]_{\mathrm{e}}$, $p_{\text {ves }}$ was strongly correlated with synaptic strength $p_{\text {ves }}\left(R^{2}=0.663\right.$, 372 Fig. 8a), while the number of readily-releasable vesicles $(M)$ showed almost no correlation $\left(R^{2}=\right.$ 373 0.018). In $4 \mathrm{mM}\left[\mathrm{Ca}^{2+}\right]_{\mathrm{e}}$, however, the impact of $N\left(R^{2}=0.471\right)$ was stronger than $p_{\text {ves }}\left(R^{2}=\right.$ 3740.271 ), suggesting that the number of readily-releasable vesicles limits the strength of a synapse 375 under conditions of high release probability (Fig. 8b). In both conditions, quantal size $q$ was not 376 correlated with synaptic strength (Fig. 8a and b). As expected, synapses with low release 377 probability showed pronounced paired-pulse facilitation ratio (Extended Data Fig. 8). The relation 378 between $p_{\text {ves }}$ and PPR followed a hyperbolic function ${ }^{29}$.
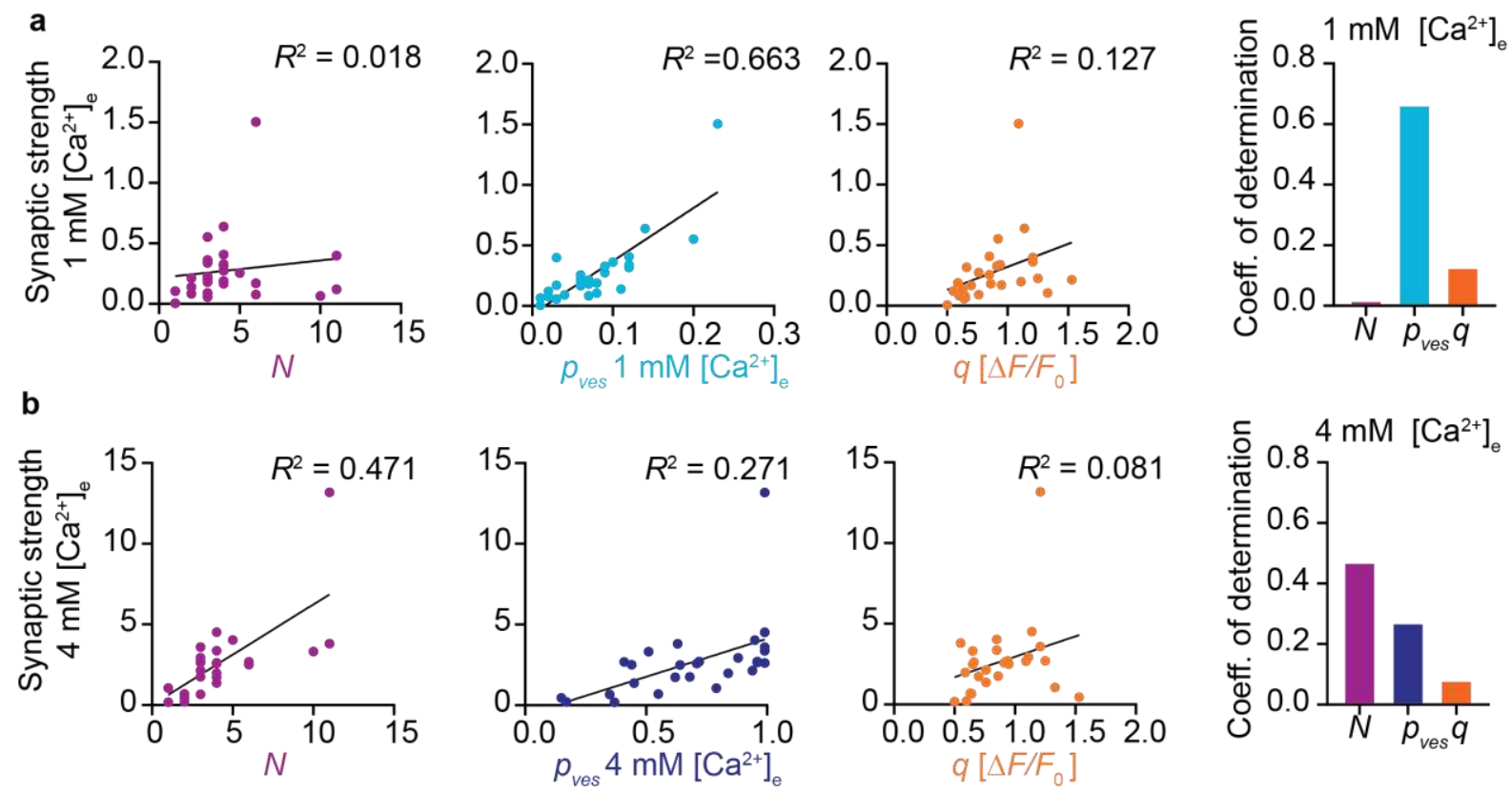

Figure 8: Quantal parameters and synaptic strength. a) Correlations between the calculated synaptic strength in 1 $\mathrm{mM}\left[\mathrm{Ca}^{2+}\right]_{\mathrm{e}}$ and the extracted quantal parameters $N, p_{\text {ves }}$ and $q(n=27$ boutons). b) Correlations between the calculated synaptic strength in $4 \mathrm{mM}\left[\mathrm{Ca}^{2+}\right]_{\mathrm{e}}$ and the extracted quantal parameters $N$, p pes and $q(n=27$ boutons). (First 3 panels) Plot of the calculated synaptic strength in $4 \mathrm{mM}\left[\mathrm{Ca}^{2+}\right]$ from the extracted quantal parameters as

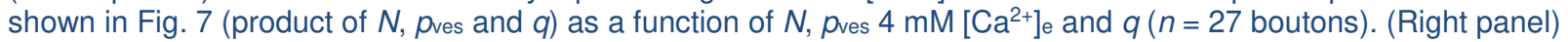
summary of the coefficient of determination for the linear correlations between synaptic strength in $4 \mathrm{mM}\left[\mathrm{Ca}^{2+}\right]_{\mathrm{e}}$ and $N\left(R^{2}=0.471\right), p_{\text {ves }}$ in $1 \mathrm{mM}\left[\mathrm{Ca}^{2+}\right]_{\mathrm{e}}\left(R^{2}=0.271\right)$ and $q\left(R^{2}=0.081\right)$.

386 In summary, most Schaffer collateral synapses are capable of increasing the glutamate output per AP under high release probability conditions and produce amplitude distributions consistent with binomial statistics. The vesicular release probability is remarkably variable between individual presynaptic terminals, suggesting that the distance between calcium channels and 
readily releasable vesicles is not uniform. Whether Schaffer collateral synapses operate in a high

391 or low release probability regime when the animal is engaged in a behavioral task and when

392 neuromodulatory inputs are active remains to be determined.

\section{Discussion}

\section{Different approaches to optical quantal analysis}

395 By measuring cleft glutamate transients at Schaffer collateral synapses under conditions of low 396 and high release probability, we directly show the impressive dynamic range of individual

397 boutons. The capacity for multivesicular release (MVR) has initially been inferred from the 398 analysis of postsynaptic currents at various synapses in the $\mathrm{CNS}^{20}$. Optical approaches to 399 quantal analysis were based on the analysis of spine $\mathrm{Ca}^{2+}$ transients (EPSCaTs) as a proxy for 400 postsynaptic depolarization ${ }^{18,30,31}$. Compared to EPSCaT measurements, glutamate imaging has 401 four distinct advantages: First, the unitary response to the release of a single vesicle (quantal 402 size $q$, here measured in units of $\Delta F / F_{0}$ ) is determined by the properties of iGluSnFR and the 403 resolution of the microscope and thus very similar across individual synapses. EPSCaT 404 amplitude, in contrast, depends on the density of NMDARs, AMPARs, and the diluting volume of 405 the spine. The unitary EPSCaT is therefore different in every synapse, and it is practically 406 impossible to wait for spontaneous EPSCaTs (the equivalent of "miniature end-plate potentials") 407 while imaging continuously. This is a serious drawback, as knowing $q$ is at the heart of a true 408 quantal analysis. Second, EPSCaTs are mediated by voltage-dependent NMDA receptors and 409 voltage-gated calcium channels. Dendritic depolarization by other active synapses can therefore 410 influence EPSCaT amplitude at the synapse under scrutiny. iGluSnFR signals, in contrast, are 411 highly localized and unlikely to be contaminated by the activity of nearby synapses. Third, 412 EPSCaTs are sensitive to the extracellular $\mathrm{Ca}^{2+}$ concentration while iGluSnFR is not. The 413 calcium-independent read-out made it possible for us to directly investigate the impact of $\left[\mathrm{Ca}^{2+}\right]_{\mathrm{e}}$ 414 on the release machinery and to replace $\mathrm{Ca}^{2+}$ by $\mathrm{Sr}^{2+}$, generating desynchronized fusion events.

415 Fourth, iGluSnFR directly probes the presynaptic function from a neuron of known identity.

416 Several boutons on the same axon can be probed in parallel or sequentially, removing the bias 417 towards strongly responding synapses that troubles EPSCaT analysis. Although other optical 418 methods, such as styryl dyes or pHluorin-based indicators, share some of the advantages of 419 iGluSnFR, they report vesicle fusion and not glutamate release. Therefore, indirect methods to 420 sense the released glutamate have been used in the past. Electrical stimulation of single 421 synapses and postsynaptic measurements of AMPAR currents has been widely used to estimate 422 the quantal properties of synaptic transmission at glutamatergic synapses in many brain areas. 
However, this method depends on faithful translation of the synaptic glutamate concentration into AMPARs currents which is measured at the soma of the postsynaptic cell. Dendritic filtering and other electrotonic effects make this analysis challenging. Furthermore, bias may be introduced by the selection of stimulation conditions which yield all-or-none responses, discarding synapses with multi-quantal response characteristics. The increase in synaptic glutamate associated with increased release probability observed in previous studies has been challenged by alternative explanations, namely diffusion of glutamate from adjacent sites $^{32}$. Could the larger glutamate concentrations we observed under high release probability conditions be a result of synaptic spillover? In contrast to extracellular field stimulation which activates a large number of axons, we triggered APs in a single CA3 pyramidal cell. As presynaptic boutons on a single active axon are spatially well separated, spillover of glutamate is extremely unlikely. As a precaution, we performed all imaging experiments at $33^{\circ} \mathrm{C}$ to ensure efficient glutamate uptake by astrocytes. Furthermore, we observed the same local confinement of iGluSnFR transient under low and high release probability conditions, indicating that the source of glutamate was solely the active zone of the bouton under investigation.

\section{Occupancy of glutamate sensors in the synaptic cleft}

440 The steady-state glutamate affinity of iGluSnFR $\left(K_{d}=2.1 \mu \mathrm{M}\right.$ on neurons $\left.{ }^{12}\right)$ is similar to neuronal AMPARs $\left(3-10 \mu \mathrm{M}^{33}\right)$. Glutamate concentrations are thought to reach $1.1 \mathrm{mM}$ in hippocampal synapses $^{34}$, but AMPAR occupancy is nevertheless quite low $^{35}$. As the glutamate transient in the synaptic cleft is very short ${ }^{36}$, the on-rate rather than the steady-state affinity determines the occupancy after vesicular glutamate release. Our Monte-Carlo simulations suggest that the saturation curves of iGluSnFR and GluA1/GluA2 heteromers in the synaptic cleft are actually quite similar. Thus, iGluSnFR is a good proxy for the activation of postsynaptic receptors, but will not linearly report cleft glutamate. To extract quantal parameters from iGluSnFR signals, it is essential to correct for indicator non-linearity, as quantal peaks will not appear at integer multiples of $q$.

As iGluSnFR competes with excitatory amino acids transporters (EAATs) for glutamate, it slows down the clearance of bulk glutamate from the extracellular space ${ }^{37}$. However, diffusion of 453 glutamate out of the synaptic cleft occurs at much shorter time scales $(<100 \mu s)$ than uptake by

454 EAATs. Our measurements of synaptic strength between pairs of connected CA3-CA1 neurons 455 showed no significant difference between iGluSnFR-expressing and non-transfected CA3 456 neurons (Extended Data Fig. 7), demonstating that buffering effects of iGluSnFR did not affect 
postsynaptic AMPAR currents. The situation might be different for global expression of iGluSnFR or during periods of dense neuronal activity.

\section{Quantal parameters and their variability}

461 Our estimate of $\sim 4$ readily releasable SVs (median) is in line with other functional

462 measurements based on statistic of synaptic transmission to quantify the number of release

463 sites $^{18,38}$. Electron tomography of glutamatergic synapses from rapidly frozen organotypic

464 hippocampal slice cultures showed 10 to 12 docked vesicles per active zone ${ }^{39}$. Thus, not all

465 docked vesicles might be release-competent. A recent study of primary hippocampal cultures

466 determined the number of distinct release sites per active zone through a clustering method ${ }^{5}$.

467 They estimated 10 release sites per active zone (assuming a release site diameter of $70 \mathrm{~nm}$ ),

468 but it is not clear whether all of these release sites are constantly occupied by release-ready

469 vesicles. Another study on dissociated neurons, using total internal reflection fluorescence

470 microscopy (TIRF) to monitor vesicle release with pHluorin, estimated 3 - 8 release sites per

471 active zone ${ }^{40}$. Thus, while the absolute number of release-ready vesicles may vary with the

472 preparation and age of the culture ${ }^{41}$, the range of 1-11 that we estimate from iGluSnFR analysis

473 is consistent with estimates from other functional imaging approaches.

475 Quantal size, the iGluSnFR fluorescence change in response to the release of a single vesicle,

476 was quite consitent between boutons ( $C V=0.29)$, with amplitudes very similar to the

477 desynchonized events we observed during $\mathrm{Sr}^{2+}$ wash-in. According to EM measurements of SV

478 diameter, the mean vesicle volume varies between individual CA1 synapses up to five-fold (5000

$479 \mathrm{~nm}^{3}$ to $25000 \mathrm{~nm}^{3}$ ) with a CV of $0.35^{42,43}$. Thus, it is possible that docked and release-ready

480 vesicles are more consistent between synapses than the total pool of SVs. There is also

481 variability of vesicle volume within boutons. Based on EM vesicle diameter measurements ${ }^{42}$, the

482 distribution of vesicle volumes is thought to be skewed with a CV $=0.38$ (Ref. $\left.{ }^{44}\right)$. The fact that

483 we and others ${ }^{45}$ observed a pronounced gap in the iGluSnFR response histogram between

484 single- and multivesicular events is not consistent with such a large variability. In our fitting

485 procedure to determine the quantal parameters, we did not account for any variability in

486 glutamate content. The only source of variability we included was photon shot noise, which we

487 could precisely determine for every bouton from the fluctuations of baseline fluorescence.

488 Including a term for quantal variability led to unrealistically broad distributions that were not 489 compatible with the multi-peaked histograms we measured in high $\mathrm{Ca}^{2+}$. Thus, it is possible that 490 only vesicles with a specific diameter or filling state can dock and fuse, which would be a novel 
491 quality control mechanism ensuring quantal uniformity ${ }^{7}$. In summary, our functional

492 measurements from live synapses suggest that glutamate quanta are more uniform than

493 ultrastructural diameter measurements would suggest.

495 The relative importance of $p_{\text {ves }}$ and $N$ depends on the state of the synapse

496 Our results show that even the smallest synapses in the brain are capable of MVR. In $1 \mathrm{mM}$

$497\left[\mathrm{Ca}^{2+}\right]_{\mathrm{e}}$, which is close to physiological $\left[\mathrm{Ca}^{2+}\right]_{\mathrm{e}}$ in awake animals ${ }^{11}$, however, pres is low $(0.01-$

498 0.23) and MVR events are quite rare. We show that under these conditions, p pres largely

499 determines synaptic strength, whereas $N$ limits synaptic output under high $p_{\text {syn }}$ conditions. In

500 vivo, where $p_{\text {syn }}$ is subject to neuromodulation, the relative impact of $p_{\text {ves }}$ and $N$ on synaptic

501 output may vary depending on the neuromodulatory state and the frequency of transmission. In

502 our dataset, $p_{\text {ves }}$ provided an excellent prediction of the paired-pulse ratio in $4 \mathrm{mM}\left[\mathrm{Ca}^{2+}\right]_{\mathrm{e}}\left(R^{2}=\right.$

503 0.91, Extended Data Fig. 8), confirming previous studies ${ }^{29,46}$. Somewhat surprisingly, the number

504 of release-ready vesicles showed no correlation with PPR, but the relatively long interval

505 between the 2 pulses (ISI = $48 \mathrm{~ms}$ ) might have been sufficient to replenish vacant release sites.

506 The number of release-ready vesicles might be rather critical for sustained transmission at high

507 frequencies ${ }^{47}$, which we did not test in this study. GluSnFR variants with faster kinetics allow

508 resolving activity during $100 \mathrm{~Hz}$ trains ${ }^{48}$, but provide fewer photons per release event. Due to its 509 excellent signal-to-noise ratio, the relatively slow iGluSnFR is a better choice for optical quantal 510 analysis at low frequencies. 


\section{Methods}

513 Slice culture preparation. Organotypic hippocampal slices were prepared from Wistar rats P5-

514 P7 as described previously ${ }^{49}$. Briefly, dissected hippocampi were cut into $400 \mu \mathrm{m}$ slices with a

515 tissue chopper and placed on a porous membrane (Millicell CM, Millipore). Cultures were

516 maintained at $37^{\circ} \mathrm{C}, 5 \% \mathrm{CO}_{2}$ in a medium containing (for $500 \mathrm{ml}$ ): $394 \mathrm{ml}$ Minimal Essential

517 Medium (Sigma M7278), $100 \mathrm{ml}$ heat inactivated donor horse serum (H1138 Sigma), $1 \mathrm{mM} \mathrm{L-}$

518 glutamine (Gibco 25030-024), $0.01 \mathrm{mg} \mathrm{ml}^{-1}$ insulin (Sigma I6634), $1.45 \mathrm{ml} 5 \mathrm{M} \mathrm{NaCl}$ (S5150

519 Sigma), $2 \mathrm{mM} \mathrm{MgSO}_{4}$ (Fluka 63126), $1.44 \mathrm{mM} \mathrm{CaCl}_{2}$ (Fluka 21114), 0.00125\% ascorbic acid

520 (Fluka 11140), 13 mM D-glucose (Fluka 49152). No antibiotics were added to the culture

521 medium. The medium was partially exchanged (60-70\%) twice per week. Wistar rats were

522 housed and bred at the University Medical Center Hamburg-Eppendorf (UKE). All procedures

523 were performed in compliance with German law (Tierschutzgesetz der Bundesrepublik

524 Deutschland, TierSchG) and according to the guidelines of Directive 2010/63/EU. Protocols

525 were approved from the Behörde für Justiz und Verbraucherschutz (BJV) -

526 Lebensmittelsicherheit und Veterinärwesen, Hamburg.

527

528 Plasmids and electroporation procedure. iGluSnFR, a gift from Loren Looger (Addgene 529 plasmid \#41732) and tdimer2, were each subcloned into a neuron-specific expression vector

$530(\mathrm{pCl})$ under the control of the human synapsin1 promoter. Plasmids were diluted to $20 \mathrm{ng} / \mu \mathrm{L}$ and $53140 \mathrm{ng} / \mu \mathrm{L}$ for tdimer2 and iGluSnFR, respectively, in K-gluconate-based solution consisting of (in $532 \mathrm{mM}$ ): $135 \mathrm{~K}$-gluconate, $4 \mathrm{MgCl}_{2}$, $4 \mathrm{Na}_{2}$-ATP, $0.4 \mathrm{Na}-\mathrm{GTP}, 10 \mathrm{Na}_{2}$-phosphocreatine, 3 ascorbate 533 and 10 HEPES (pH 7.2). CA3 pyramidal neurons were transfected by single cell electroporation 534 between DIV 17 and DIV 25 with a mixture of the two plasmids. During the electroporation

535 procedure, slice cultures were maintained in pre-warmed HEPES-buffered solution consisting of 536 (in mM): $145 \mathrm{NaCl}, 10 \mathrm{HEPES}, 25 \mathrm{D}$-glucose, $1 \mathrm{MgCl}_{2}$ and $2 \mathrm{CaCl}_{2}$ (pH 7.4, sterile filtered). An 537 Axoporator 800A (Molecular Devices) was used to deliver 50 voltage pulses (-12 V, $0.5 \mathrm{~ms})$ at $53850 \mathrm{~Hz}^{50}$.

539

540 Solutions and Electrophysiology. Experiments were performed 2 to 4 days after

541 electroporation. Hippocampal slice cultures were placed in the recording chamber of the 542 microscope and superfused with artificial cerebrospinal fluid (ACSF) containing (in mM): 127

$543 \mathrm{NaCl}, 25 \mathrm{NaHCO}_{3}, 25 \mathrm{D}$-glucose, $1.25 \mathrm{NaH}_{2} \mathrm{PO}_{4}, 2.5 \mathrm{KCl}, 2 \mathrm{CaCl}_{2}, 1 \mathrm{MgCl}_{2}$. ACSF was

544 saturated with $95 \% \mathrm{O}_{2}$ and $5 \% \mathrm{CO}_{2}$. In the experiments where $\left[\mathrm{Ca}^{2+}\right]_{\mathrm{e}}$ was changed, we 545 switched from $1 \mathrm{mM} \mathrm{Ca}^{2+}, 4 \mathrm{mM} \mathrm{Mg}^{2+}$ to $4 \mathrm{mM} \mathrm{Ca}^{2+}, 1 \mathrm{mM} \mathrm{Mg}^{2+}$ to keep the divalent ion 
546 concentration constant. Patch pipettes with a tip resistance of 3.5 to $4.5 \mathrm{M} \Omega$ were filled with (in $547 \mathrm{mM}$ ): $135 \mathrm{~K}$-gluconate, $4 \mathrm{MgCl}_{2}, 4 \mathrm{Na}_{2}$-ATP, $0.4 \mathrm{Na}$-GTP, $10 \mathrm{Na}_{2}$-phosphocreatine, 3 ascorbate 548 and 10 Hepes ( $\mathrm{pH} 7.2$ ). Experiments were performed at $33^{\circ} \mathrm{C} \pm 1^{\circ} \mathrm{C}$ by controlling the 549 temperature of the ACSF with an in-line heating system and the oil immersion condenser with a 550 Peltier element. Whole-cell recordings from transfected CA3 pyramidal neurons were made with 551 a Multiclamp 700B amplifier (Molecular Devices) under the control of Ephus software written in 552 Matlab ${ }^{51}$. CA3 neurons were held in current clamp and stimulated through the patch pipette by 553 brief electrical pulses (2-3 ms, 1.5-3.5 nA) to induce single APs. Individual trials (single pulse or 554 paired-pulse) were delivered at a frequency of $0.1 \mathrm{~Hz}$. The analog signals were filtered at $6 \mathrm{kHz}$ 555 and digitized at $10 \mathrm{kHz}$. For dual patch experiments, CA1 neurons were recorded in voltage 556 clamp. Access resistance $\left(\mathrm{R}_{\mathrm{acc}}\right)$ was monitored continuously throughout the experiment and 557 recordings with $\mathrm{R}_{\mathrm{acc}}>20 \mathrm{M} \Omega$ were discarded. To isolate AMPA receptor responses, $10 \mu \mathrm{M}$ CPP558 ene was added to the perfusate.

559 For extracellular synaptic stimulation, a monopolar electrode was placed in stratum radiatum and 560 two $0.2 \mathrm{~ms}$ pulses, $48 \mathrm{~ms}$ apart, were delivered using an ISO-Flex stimulator (A.M.P.I.).

561 Stimulation intensity was adjusted to be subthreshold for APs. $10 \mathrm{mM} \gamma$-DGG was added to the

562 bath in experiments were the fold change in AMPARs was probed under decrease of AMPARs 563 saturation.

564

565 Two-photon microscopy. The custom-built two-photon imaging setup was based on an

566 Olympus BX51WI microscope controlled by a customized version the open-source software 567 package Scanlmage ${ }^{13}$ written in MATLAB (MathWorks). We used a pulsed Ti:Sapphire laser 568 (MaiTai DeepSee, Spectra Physics) tuned to $980 \mathrm{~nm}$ to simultaneously excite both the 569 cytoplasmic tdimer2 and the membrane bound iGluSnFR. Red and green fluorescence was 570 detected through the objective (LUMPLFLN 60XW, 60×, 1.0 NA, Olympus) and through the oil 571 immersion condenser (1.4 NA, Olympus) using 2 pairs of photomultiplier tubes (PMTs, H7422P572 40SEL, Hamamatsu) (Extended Data Fig. 1), 560 DXCR dichroic mirrors and 525/50 and 607/70 573 emission filters (Chroma Technology) were used to separate green and red fluorescence.

574 Excitation light was blocked by short-pass filters (ET700SP-2P, Chroma). Scanlmage was 575 modified to allow arbitrary line scanning. To measure iGluSnFR signals with a high signal-to576 noise ratio, spiral scans were acquired to sample the surface of individual boutons. For single 577 pulse stimulation, we acquired 44 spiral lines at $500 \mathrm{~Hz}$ or $330 \mathrm{~Hz}$. For paired-pulse pulse 578 stimulation (48 ms ISI), we acquired 64 spiral lines at $500 \mathrm{~Hz}$. Photomultiplier dark noise was 579 measured before shutter opening and subtracted for every trial. 
Drift Correction. To compensate for movements of the tissue during long imaging sessions, we developed an automated drift correction algorithm to re-center the synapse of interest. As spatial reference, we obtained a series of optical sections (z-step size: $0.5 \mu \mathrm{m}$ ) that were interpolated to $0.25 \mu \mathrm{m}$. For drift correction, we acquired a single frame-scan (test image) and performed subpixel image registration against the stack of reference images to extract lateral drift. In a second step, the overlapping regions from both, the test image and reference images were compared via cross correlation to reveal axial drift. Drift was compensated by adding offsets to the xy-scanner command voltages and by moving the objective to the correct z-position. Drift correction was typically completed within $0.3 \mathrm{~s}$ and performed before each stimulation trial.

Analysis of fluorescence transients. In case of a release event ('success'), a spiral scan covering the entire bouton may hit the diffusing cloud of glutamate just once or several times per line. We had no prior knowledge about the precise location of fusion events on the bouton surface. To maximize the signal-to-noise ratio in every trial, we assigned a dynamic region of interest (ROI): Pixel columns (i.e. spatial positions) were sorted according to the change in fluorescence $(\Delta F)$ in each column. In 'success' trials (average $\Delta F>2 \sigma$ of baseline noise), only columns which displayed a clear change in fluorescence $(\Delta F>1 / 2 \max (\Delta F))$ were evaluated. In 'failure' trials (when $\Delta F$ of each column of the $\mathrm{ROI}$ was $5 \%>$ than $\Delta F$ of the corresponding columns in the baseline), the columns selected in the last 'success' trial were evaluated. At that stage, the classification used for ROI positioning (success vs. failure) was preliminary. Indeed, some 'failure' trials did show small fluorescent transients in the more sensitive ROI-based analysis. Boutons with a full width at half maximum (FWHM) of the amplitude distribution of the baseline (i.e. non-stimulated trials) larger than 0.4 were rejected as the imaging conditions were considered non-optimal and not considered for further analysis. To correct for bleaching, we fit an exponential decay to $F_{0}$ in failure trials. We corrected all data for bleaching by subtracting monoexponential fits acquired from the average fluorescence time course of failures. This bleach time constant was used to establish a photobleaching correction for each trial. To measure the amplitude iGluSnFR changes in fluorescence and to distinguish successful release of glutamate from failures, we used a template-based fitting algorithm. For each bouton we extracted a characteristic decay time constant by fitting a mono-exponential function to the 611 every trial we kept the amplitude as the only free parameter. To check for overall stability, we 612 measured the mean $F_{0}$ (Baseline iGluSnFR signal) in each trial. If a trial had a $F_{0}>2 \sigma$ measured 613 out of the average $F_{0}$ of all trials then that trial was removed from the analysis. 
614 The number of successes divided by the number of trials is the probability of release for a

615 synapse $\left(p_{\text {syn }}\right)$. We define the success amplitude to a single stimulus as the cleft glutamate

616 ([Glu $\left.]_{\text {success }}\right)$.

617

618 Localization of release events. To map the position of fusion events on a bouton, we acquired

61915 frames (16 × 16 pixels) at a rate of $62.5 \mathrm{~Hz}$ (16 ms per frame). Analysis of data consisted of

620 four steps: de-noising, image alignment, estimating the amplitude of fluorescence transients, and

621 localization of the release site. Raw images were first treated by a wavelet method to reduce

622 photon shot noise ${ }^{52}$. Next, a cross talk correction was applied and the images were smoothed

623 using a low pass filter (Gaussian kernel, $5 \times 5$ field size, $\sigma=1$ pixel). Images were then up-

624 sampled to $128 \times 128$ pixels (Lanczos3 kernel). For image alignment, we performed a Fast

625 Fourier transform (FFT) on the red fluorescence signal (tdimer2). In addition, we used the red

626 channel to define a continuous area encompassing bouton and axon (pixel intensity $\geq 10 \%$ to

$62730 \%$ of maximal intensity) as a morphology mask. The relative change in iGluSnFR fluorescence

$628\left(\Delta F / F_{0}\right)$ was calculated using the mean of 5 baseline frames as $F_{0}$ (pixel by pixel). The top $3 \%$ of

629 pixel values within the bouton mask were averaged to get the peak amplitude. All trials were

630 tested for baseline stability which was defined as having overall highly similar images throughout

631 the baseline frames (cross correlation $\geq 0.9$ ). Trials not passing the threshold, for example when

632 green fluorescent particles happened to pass through the bouton of interest, were excluded from

633 further analysis.

634 To localize the fusion site, we constructed a template (2-D anisotropic Gaussian kernel) from the

635 average of 5 'success' trials. In the first round of analysis, we fit the template to every signal

636 frame by adapting only the amplitude, keeping the location and shape of the kernel fixed at the

637 template values. In trials where the release site is located not exactly at the template position,

638 this procedure will underestimate the true amplitude. The goal of this first pass analysis was a

639 preliminary classification of 'successes' $\left(\Delta F / F_{0}>2 \sigma\right.$ of baseline noise) and 'failures' $\left(\Delta F / F_{0}<2 \sigma\right.$

640 of baseline noise). To localize the fusion site in each individual trial, the fitting procedure was

641 repeated, this time allowing for variable location. The location of the best fit corresponded to the

642 most likely fusion site. This analysis was applied to all success trials, and a probability density

643 function (2D Gaussian) was fitted to estimate the size of the active zone on the bouton. The

644 same localization procedure was applied to the failure trials and, as a control, to a frame before

645 stimulation. In most experiments, these two datasets produced random localizations, suggesting

646 that our $2 \sigma$ criterion was suitable to distinguish successes from failures. In cases where the

647 positions of apparent 'failures' clustered in a second area of the bouton, we classified this bouton

648 as 'multi-synapse bouton' (Extended Data Fig. 4) and excluded it from further analysis. 
649 To estimate the precision of our localization procedure, we imaged carboxylate-modified yellow-

650 green fluorescent microbeads $(0.17 \mu \mathrm{m}$ diameter) positioned next to red fluorescent boutons and 651 used the localization procedure described above to determine bead position relative to the 652 bouton. As expected, localization precision was a function of the number of photons detected 653 from the bead. At low laser power, we could match the bead intensity to the typical signal 654 amplitude of iGluSnFR to the release of a single vesicle. Under these conditions, bead 655 localization precision was $0.005 \mu \mathrm{m} \pm 0.01 \mu \mathrm{m}$.

657 Synapse modeling and glutamate release simulation. Release of glutamate and the time 658 profile of iGluSnFR fluorescence were simulated using a Monte Carlo method (MCell) that 659 considers the stochastic nature of molecule diffusion as well as that of reaction between 660 molecules. The model consisted of an axon (diameter $0.2 \mu \mathrm{m}$, length $3 \mu \mathrm{m}$ ) with a varicosity 661 representing the bouton (diameter $0.5 \mu \mathrm{m}$, length $0.5 \mu \mathrm{m}$ ), a hemispheric structure representing 662 the spine (diameter $0.4 \mu \mathrm{m}$ ) attached to a cylindrical spine neck (diameter $0.2 \mu \mathrm{m}$ ). Active zone 663 and postsynaptic density were defined as circular areas (diameter $300 \mathrm{~nm}$ ) separated by the 664 synaptic cleft $(20 \mathrm{~nm})^{53}$. Axon and spine were enclosed by an astrocytic compartment (width of 665 extracellular space: $20 \mathrm{~nm}$ ). Boundary conditions for the entire system were reflective.

666 Glutamate transporters (GluT) were placed on astrocytic membranes at a density of $10.000 \mu^{-}$

6672 . AMPARs were restricted to the PSD at a density of $1.200 \mu^{-2}$ (resulting in $\sim 85$ receptors at a 668 PSD diameter of $300 \mathrm{~nm}$ ). Vesicle fusion was modeled by an instantaneous injection of 669 glutamate at a fixed position (center of the active zone). The glutamate content of a single 670 vesicle was estimated to be in the range of 2.000-3.000 molecules ${ }^{25}$. The diffusion coefficient of 671 glutamate ${ }^{54}$ was set to $200 \mu \mathrm{m}^{2} / \mathrm{s}$. To study the consequences of univesicular and multivesicular 672 release, we varied the number of released glutamate molecules between 3000 and 30.000 (1-10 673 vesicles).

674 Our model of iGluSnFR is based on a two-step reaction where rapid binding of glutamate to 675 iGluSnFR is followed by a slower conformational change with concomitant increase in 676 fluorescence. The kinetics for step 2 is different for purified protein vs. cell-based 677 measurements ${ }^{8}$. We therefore measured iGluSnFR fluorescence time profiles following vesicle 678 release at a functional bouton by parking the imaging laser beam at a fixed position (Extended 679 Data Fig. 9). The rise time of the $\Delta F / F_{0}$ profile was fitted to a model in which two irreversible 680 consecutive first-order reactions are linked:

$$
\text { Glu }+ \text { iGluSnFR } \rightarrow \text { glu-iGluSnFR }{ }_{\text {dim }} \rightarrow \text { glu-iGluSnFR' }{ }_{\text {bright }}
$$

682 Given the fast rate $k_{-1}$ re-starting this reaction (conformational change) is less likely than 683 dissociation of glutamate from iGluSnFR' bright. In addition, the high diffusion constant of 
684 glutamate has led to a drop of glutamate concentration at the release site back to the

685 background level and no new glu-iGluSnFR will form that otherwise would affect the measured

686 fluorescence. Hereby, we get values $k_{+2}$ of $2.48 \times 10^{3} \mathrm{M}^{-1} \mathrm{~s}^{-1}$ and $k_{-2}$ of $111 \mathrm{~s}^{-1}$ for the

687 conformational change (Extended Data Fig. 9). We used a kinetic AMPA receptor model ${ }^{26}$ based $^{2}$

688 on recordings $\left(22-24^{\circ} \mathrm{C}\right)$ from rat hippocampal slices (age: p15 - p24). This model represents

689 GluA1/GluA2 heteromers that are typical for CA3-CA1 synapses. We adjusted the rate constants

690 to match the temperature of our experiments $\left(33^{\circ} \mathrm{C}\right)$.

691

692 Quantal analysis. We wrote custom code (Matlab) to extract quantal parameters. Analysis of

693 optical signals is fundamentally different from EPSP analysis as the sources of noise are

694 different and indicator saturation has to be considered. When collecting (green) fluorescence,

695 the photon 'shot noise' follows Poisson statistics. For each bouton, we measured the standard

696 deviation of baseline fluorescence $(\sigma)$ in each trial before stimulation (baseline noise). Imaging

697 conditions varied between individual experiments (depth in tissue, expression level, laser power)

698 and we discarded experiments with baseline noise above $40 \%$. For the remaining boutons, we

699 generated predicted amplitude distributions based on binomial statistics. The width of the

700 success distributions (Gaussians) was determined by the expected photon shot noise calculated

701 from the baseline noise. Propagation of the shot noise was considered in quadrature:

702

703

704

705

706

707

Statistical Analysis. Normality was tested using D'Agostino-Pearson omnibus normality test. To

708 test for significant differences between population means, Paired t test or the nonparametric

709 Wilcoxon-Signed rank test. For independent population we used unpaired t test or the nonparametric Mann-Whitney test as appropriate. Statistical significance was assumed when $p$ $<0.05$. Symbols used for assigning significance in figures: not significant (n.s.), $p>0.05$;

712 significant, $p<0.05\left(^{*}\right), p<0.01\left(^{* *}\right)$, and $p<0.001\left(^{* * *}\right)$.

\section{Acknowledgements}

715 We thank Christine E. Gee and Mauro Pulin for critical reading of the manuscript and Iris Ohmert 716 and Sabine Graf for slice culture preparation and excellent technical support. We thank Gary S.

717 Bhumbra for helpful advice about quantal parameter extraction. This study was supported by the 
718 Deutsche Forschungsgemeinschaft (DFG) through Priority Programme SPP 1665 (to TGO) \& 7191926 (to JSW), Collaborative Research Center SFB 936 (to TGO), Research Unit FOR 2419 (to 720 TGO \& JSW), and by the Landesforschungsförderung Hamburg (Z-FR LF) (to TGO).

\section{Author contributions}

722 C.D.D., J.S.W., and T.G.O. designed the experiments. C.D.D. and T.G.O. prepared the 723 manuscript. C.D.D. performed synaptic imaging experiments and analyzed the data. C.S. wrote 724 software to acquire and analyze GEGI data. N.H. and K.T. provided kinetic measurements of 725 iGluSnFR.

\section{Competing financial interests}

727 The authors declare no competing interest.

728 


\section{References}

1. Del Castillo, J. \& Katz, B. Quantal components of the end-plate potential. J Physiol 124, 560-573 (1954).

2. Redman, S. Quantal analysis of synaptic potentials in neurons of the central nervous system. Physiol. Rev. 70, 165-198 (1990).

3. Mainen, Z. F., Malinow, R. \& Svoboda, K. Synaptic calcium transients in single spines indicate that NMDA receptors are not saturated. Nature 399, 151-155 (1999).

4. Nimchinsky, E. A., Yasuda, R., Oertner, T. G. \& Svoboda, K. The number of glutamate receptors opened by synaptic stimulation in single hippocampal spines. J Neurosci 24, 2054-2064 (2004).

5. Maschi, D. \& Klyachko, V. A. Spatiotemporal Regulation of Synaptic Vesicle Fusion Sites in Central Synapses. Neuron 94, 65-73.e3 (2017).

6. Tang, A.-H. et al. A trans-synaptic nanocolumn aligns neurotransmitter release to receptors. Nature 536, 210-4 (2016).

7. Rost, B. R. et al. Optogenetic acidification of synaptic vesicles and lysosomes. Nat. Neurosci. 18, 1845-1852 (2015).

8. Marvin, J. S. et al. An optimized fluorescent probe for visualizing glutamate neurotransmission. Nat. Methods 10, 162-170 (2013).

9. Dürst, C. D. et al. High-speed imaging of glutamate release with genetically encoded sensors. Nat. Protoc. 14, 1401-1424 (2019).

10. Jensen, T. P. et al. Multiplex imaging relates quantal glutamate release to presynaptic Ca2+ homeostasis at multiple synapses in situ. Nat. Commun. 10, 1414 (2019).

11. Ding, F. et al. Changes in the composition of brain interstitial ions control the sleep-wake cycle. Science (80-. ). 352, 550-555 (2016).

12. Marvin, J. S. et al. Stability, affinity, and chromatic variants of the glutamate sensor iGluSnFR. Nat. Methods 15, 936-939 (2018).

13. Pologruto, T. A., Sabatini, B. L. \& Svoboda, K. Scanlmage: Flexible software for operating laser scanning microscopes. Biomed. Eng. Online 2, 13 (2003).

14. Christie, J. M. \& Jahr, C. E. Multivesicular release at Schaffer collateral-CA1 hippocampal synapses. J. Neurosci. 26, 210-216 (2006).

15. Bolshakov, V. Y., Golan, H., Kandel, E. R. \& Siegelbaum, S. A. Recruitment of new sites of synaptic transmission during the CAMP- dependent late phase of LTP at CA3-CA1 synapses in the hippocampus. Neuron 19, 635-651 (1997).

16. Boucher, J., Kröger, H. \& Sík, A. Realistic modelling of receptor activation in hippocampal excitatory synapses: Analysis of multivesicular release, release location, temperature and synaptic cross-talk. Brain Struct. Funct. 215, 49-65 (2010).

17. Ricci-Tersenghi, F., Minneci, F., Sola, E., Cherubini, E. \& Maggi, L. Multivesicular Release at Developing Schaffer Collateral-CA1 Synapses: An Analytic Approach to Describe Experimental Data. J. Neurophysiol. 96, 15-26 (2006).

18. Oertner, T. G., Sabatini, B. L., Nimchinsky, E. A. \& Svoboda, K. Facilitation at single synapses probed with optical quantal analysis. Nat. Neurosci. 5, 657-64 (2002). 
19. Tong, G. \& Jahr, C. E. Multivesicular release from excitatory synapses of cultered hippocampal neurons. Neuron 12, 51-59 (1994).

20. Rudolph, S., Tsai, M.-C., von Gersdorff, H. \& Wadiche, J. I. The ubiquitous nature of multivesicular release. Trends Neurosci. 38, 428-38 (2015).

21. Goda, Y. \& Stevens, C. F. Two components of transmitter release at a central synapse. Proc. Natl. Acad. Sci. U. S. A. 91, 12942-12946 (1994).

22. Evans, C. S., Ruhl, D. a. \& Chapman, E. R. An Engineered Metal Sensor Tunes the Kinetics of Synaptic Transmission. J. Neurosci. 35, 11769-11779 (2015).

23. Xu-Friedman, M. A. \& Regehr, W. G. Probing fundamental aspects of synaptic transmission with strontium. J. Neurosci. 20, 4414-4422 (2000).

24. Blumer, C. et al. Automated analysis of spine dynamics on live CA1 pyramidal cells. Med. Image Anal. 19, 87-97 (2015).

25. Rusakov, D. A., Savtchenko, L. P., Zheng, K. \& Henley, J. M. Shaping the synaptic signal: Molecular mobility inside and outside the cleft. Trends Neurosci. 34, 359-369 (2011).

26. Jonas, P., Major, G. \& Sakmann, B. Quantal components of unitary EPSCs at the mossy fibre synapse on CA3 pyramidal cells of rat hippocampus. J. Physiol. 472, 615-63 (1993).

27. O'Connor, D. H., Wittenberg, G. M. \& Wang, S. S.-H. Timing and contributions of presynaptic and post-synaptic parameter changes during unitary plasticity events at CA3CA1 synapses. Synapse 61, 664-678 (2007).

28. Boyd, I. A. \& Martin, A. R. The end-plate potential in mammalian muscle. J. Physiol. 132, 74-91 (1956).

29. Debanne, D., Guerineau, N. C., Gahwiler, B. H. \& Thompson, S. M. Paired-pulse facilitation and depression at unitary synapses in rat hippocampus: quantal fluctuation affects subsequent release. J Physiol 491 ( Pt 1, 163-176 (1996).

30. Emptage, N. J., Reid, C. a, Fine, A. \& Bliss, T. V. . Optical Quantal Analysis Reveals a Presynaptic Component of LTP at Hippocampal Schaffer-Associational Synapses. Neuron 38, 797-804 (2003).

31. Yuste, R., Majewska, A., Cash, S. S. \& Denk, W. Mechanisms of calcium influx into Hippocampal spines: Heterogeneity among spines, coincidence detection by NMDA receptors, and optical quantal analysis. J Neurosci 19, 1976-1987 (1999).

32. Barbour, B. \& Häusser, M. Intersynaptic diffusion of neurotransmitter. Trends Neurosci. 20, 377-384 (1997).

33. Kessler, M., Suzuki, E., Montgomery, K. \& Arai, A. C. Physiological significance of highand low-affinity agonist binding to neuronal and recombinant AMPA receptors. Neurochem. Int. 52, 1383-1393 (2008).

34. Frerking, M. \& Wilson, M. Saturation of postsynaptic receptors at central synapses? Curr. Opin. Neurobiol. 6, 395-403 (1996).

35. McAllister, A. K. \& Stevens, C. F. Nonsaturation of AMPA and NMDA receptors at hippocampal synapses. Proc Natl Acad Sci U S A 97, 6173-6178 (2000).

36. Scimemi, A. \& Beato, M. Determining the neurotransmitter concentration profile at active synapses. Mol. Neurobiol. 40, 289-306 (2009).

37. Armbruster, M., Dulla, C. G. \& Diamond, J. S. Effects of fluorescent glutamate indicators on neurotransmitter diffusion and uptake. Elife 9, 1-26 (2020). 
38. Siksou, L. et al. A common molecular basis for membrane docking and functional priming of synaptic vesicles. Eur. J. Neurosci. 30, 49-56 (2009).

39. Imig, C. et al. The Morphological and Molecular Nature of Synaptic Vesicle Priming at Presynaptic Active Zones. Neuron 84, 416-431 (2014).

40. Funahashi, J., Tanaka, H. \& Hirano, T. Visualization of Synchronous or Asynchronous Release of Single Synaptic Vesicle in Active-Zone-Like Membrane Formed on NeuroliginCoated Glass Surface. Front. Cell. Neurosci. 12, 1-10 (2018).

41. Rose, T., Schoenenberger, P., Jezek, K. \& Oertner, T. G. Developmental refinement of vesicle cycling at Schaffer collateral synapses. Neuron 77, 1109-21 (2013).

42. Qu, L., Akbergenova, Y., Hu, Y. \& Schikorski, T. Synapse-to-synapse variation in mean synaptic vesicle size and its relationship with synaptic morphology and function. J. Comp. Neurol. 514, 343-352 (2009).

43. Hu, Y., Qu, L. \& Schikorski, T. Mean synaptic vesicle size varies among individual excitatory hippocampal synapses. Synapse 62, 953-957 (2008).

44. Soares, C., Trotter, D., Longtin, A., Béïque, J.-C. \& Naud, R. Parsing Out the Variability of Transmission at Central Synapses Using Optical Quantal Analysis. Front. Synaptic Neurosci. 11, 22 (2019).

45. James, B., Darnet, L., Moya-Díaz, J., Seibel, S.-H. \& Lagnado, L. An amplitude code transmits information at a visual synapse. Nat. Neurosci. 22, 1140-1147 (2019).

46. Sakamoto, H. et al. Synaptic weight set by Munc13-1 supramolecular assemblies. Nat. Neurosci. 21, 41-55 (2018).

47. Pulido, C., Trigo, F. F., Llano, I. \& Marty, A. Vesicular Release Statistics and Unitary Postsynaptic Current at Single GABAergic Synapses. Neuron 85, 159-172 (2014).

48. Helassa, N. et al. Ultrafast glutamate sensors resolve high-frequency release at Schaffer collateral synapses. Proc. Natl. Acad. Sci. 115, 5594-5599 (2018).

49. Gee, C. E., Ohmert, I., Wiegert, J. S. \& Oertner, T. G. Preparation of slice cultures from rodent hippocampus. Cold Spring Harb. Protoc. 2017, 126-130 (2017).

50. Wiegert, J. S., Gee, C. E. \& Oertner, T. G. Single-cell electroporation of neurons. Cold Spring Harb. Protoc. 2017, 135-138 (2017).

51. Suter, B. A. et al. Ephus: multipurpose data acquisition software for neuroscience experiments. Front. Neural Circuits 4, 1-12 (2010).

52. Tigaret, C. M., Tsaneva-Atanasova, K., Collingridge, G. L. \& Mellor, J. R. Wavelet transform-based de-noising for two-photon imaging of synaptic $\mathrm{Ca2}+$ transients. Biophys. J. 104, 1006-1017 (2013).

53. Mishchenko, Y. et al. Ultrastructural analysis of hippocampal neuropil from the connectomics perspective. Neuron 67, 1009-1020 (2010).

54. Nielsen, T. A., DiGregorio, D. A. \& Silver, R. A. Modulation of glutamate mobility reveals the mechanism underlying slow-rising AMPAR EPSCs and the diffusion coefficient in the synaptic cleft. Neuron 42, 757-771 (2004). 


\section{Figures}

a

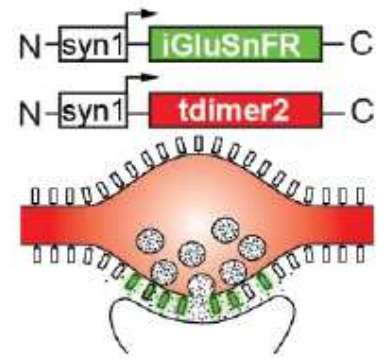

b

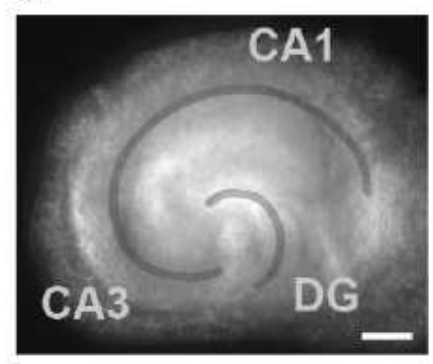

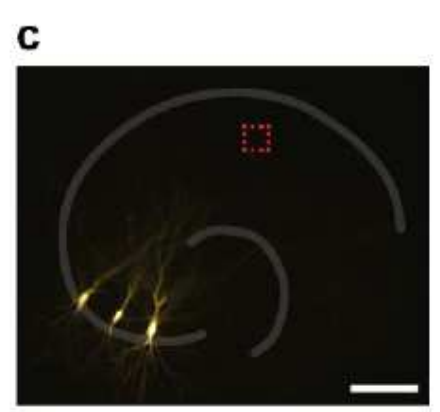
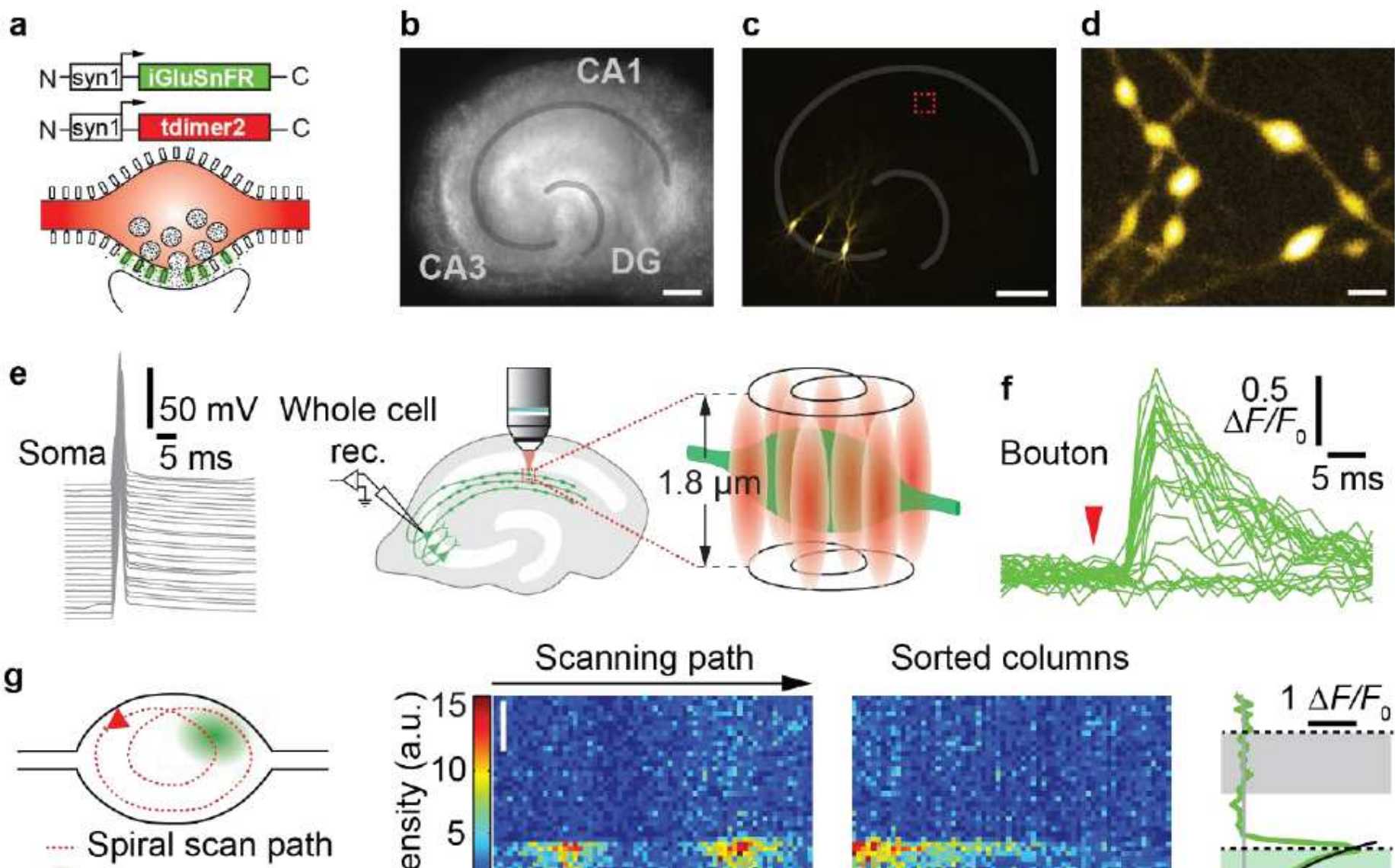
Glutamate release
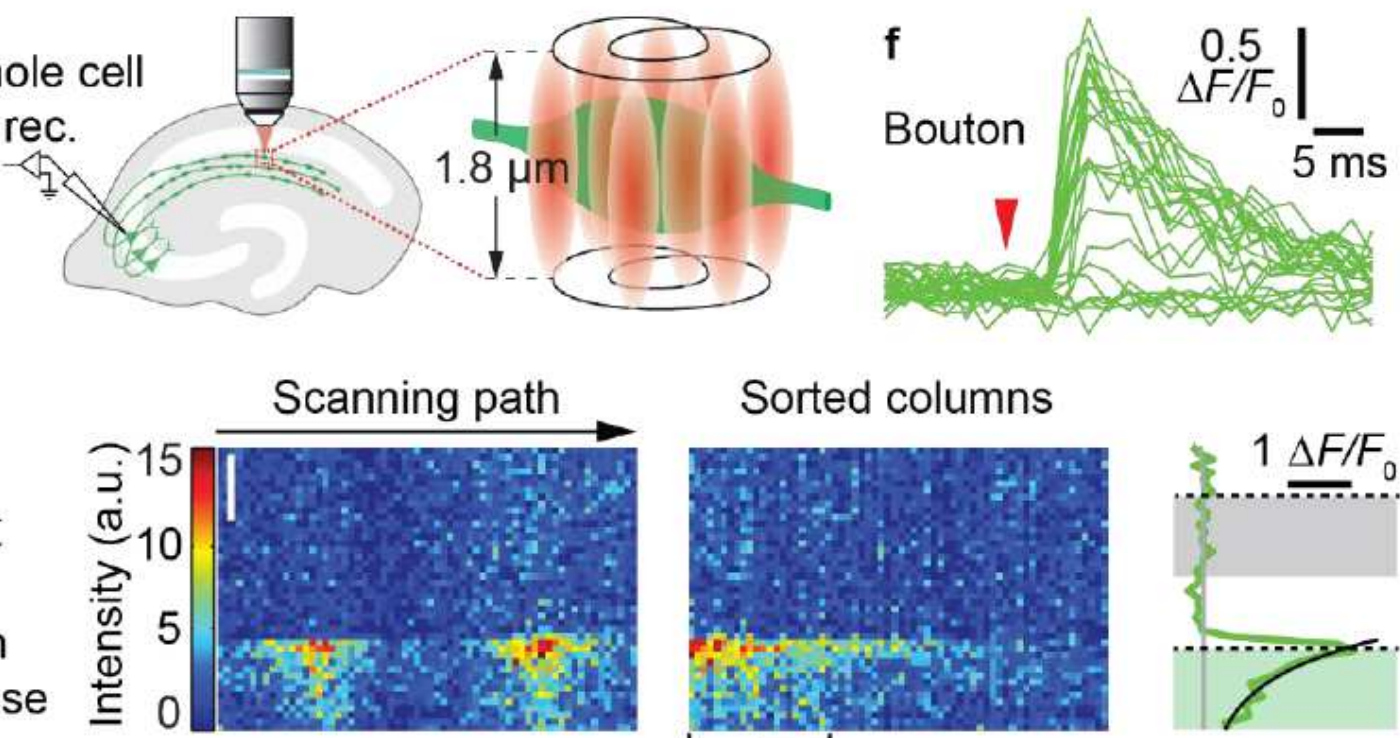

Sorted columns
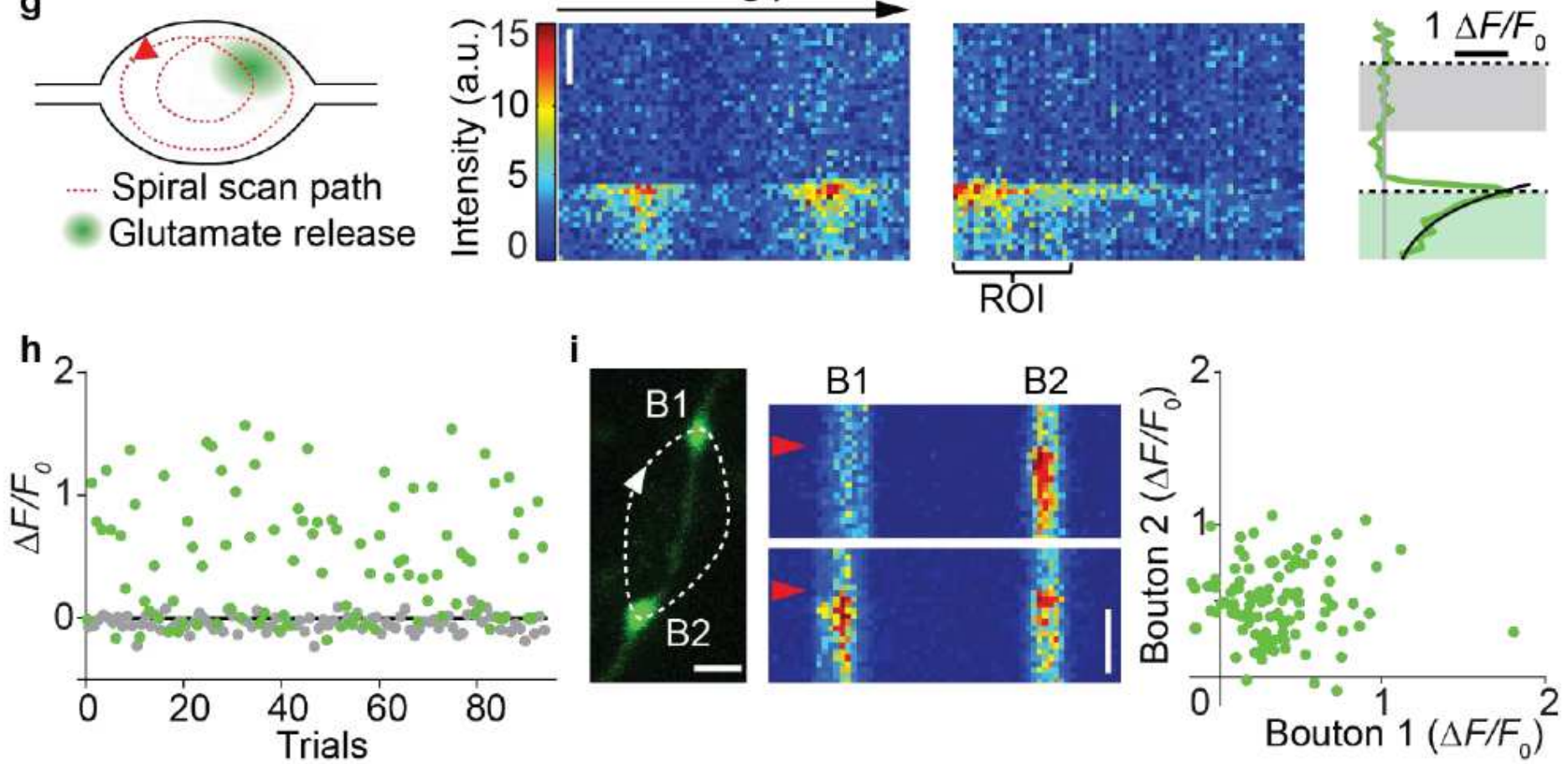

\section{Figure 1}

iGluSnFR expression in CA3 pyramidal cells of organotypic slice culture of rat hippocampus. a) En passant bouton co-expressing the cytoplasmic red fluorescent protein tdimer2 and green iGluSnFR exposed to the extracellular space. b) Transmitted light image of transfected organotypic hippocampal culture. Scale bar, $500 \mu \mathrm{m}$. c) Fluorescence image of three transfected CA3 neurons. Area for synaptic imaging is indicated (dotted box). Scale bar, $500 \mu \mathrm{m}$. d) Maximum intensity projection of a two-photon image stack of CA3 axons and their en passant boutons co-expressing tdimer2 and iGluSnFR in CA1 
stratum radiatum. Scale bar, $1 \mu \mathrm{m}$. e) APs were reliably elicited in a transfected neuron by somatic current injections while glutamate release was simultaneously imaged from a single Schaffer collateral bouton in CA1. Owing to the elongated point-spread function (PSF; red oval objects), the upper and lower surface of a bouton were simultaneously sampled by $500 \mathrm{~Hz}$ spiral scanning. f) Optical recording (iGluSnFR fluorescence) from a single Schaffer collateral bouton in CA1, showing a broad distribution of amplitudes and occasional failures. Red arrowhead indicates somatic current injection in CA3. g) Release events were typically sampled twice during every spiral scan. Fluorescence intensity (single trial) coded in rainbow colors. At $\mathrm{t}=58 \mathrm{~ms}$, a glutamate release event occurred. Scale bar, $20 \mathrm{~ms}$. Only columns with $\Delta \mathrm{F}$ $>1 / 2 \max (\Delta \mathrm{F})$ were analyzed (dynamic region-of-interest). Response amplitude was estimated by exponential fit (green area, black fit) normalized by the resting fluorescence of the bouton (gray area, grey fit). The intersection between the horizontal black dashed lines and the fits corresponds to the extracted amplitudes (average peak amplitude for each experiment). h) Response amplitude (green circles) was constant over time. A time window before stimulation was analyzed to estimate imaging noise (gray circles). Response amplitudes were monitored in ACSF containing $2 \mathrm{mM}[\mathrm{Ca} 2+]$ e at $33^{\circ} \mathrm{C}$. i) Monitoring two neighboring boutons located on the same axon. Scale bar, $1 \mu \mathrm{m}$. Two trials showing independent release events. Red arrowhead indicates the stimulation onset. Scale bar, $20 \mathrm{~ms}$. iGluSnFR transients between neighboring boutons were not correlated $(\mathrm{R} 2<0.0005)$. 
a - Success Failures Baseline

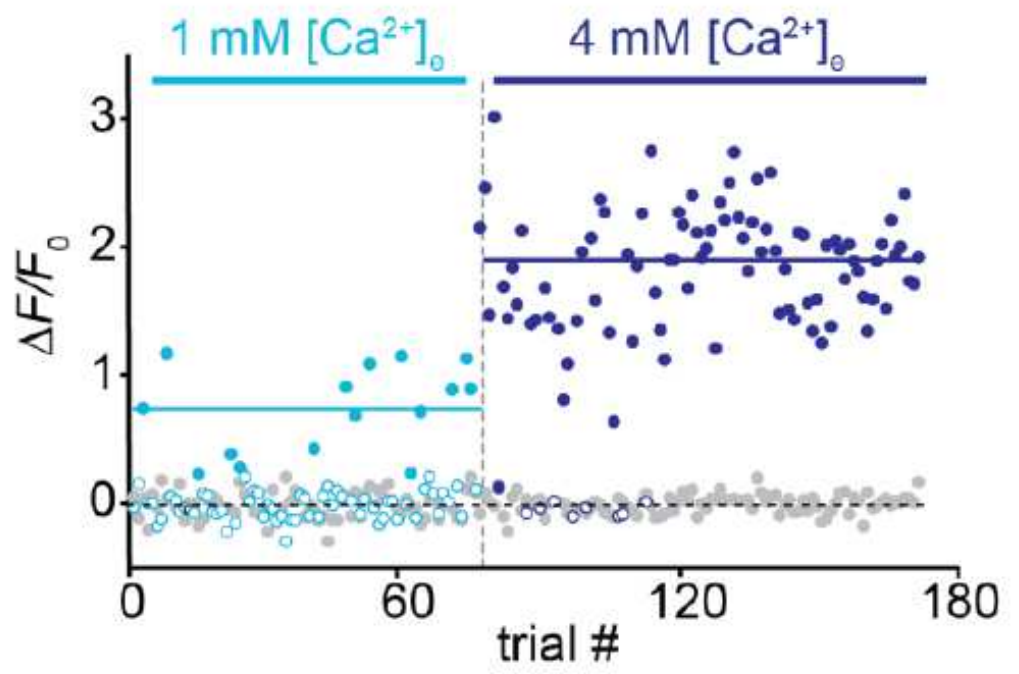

C

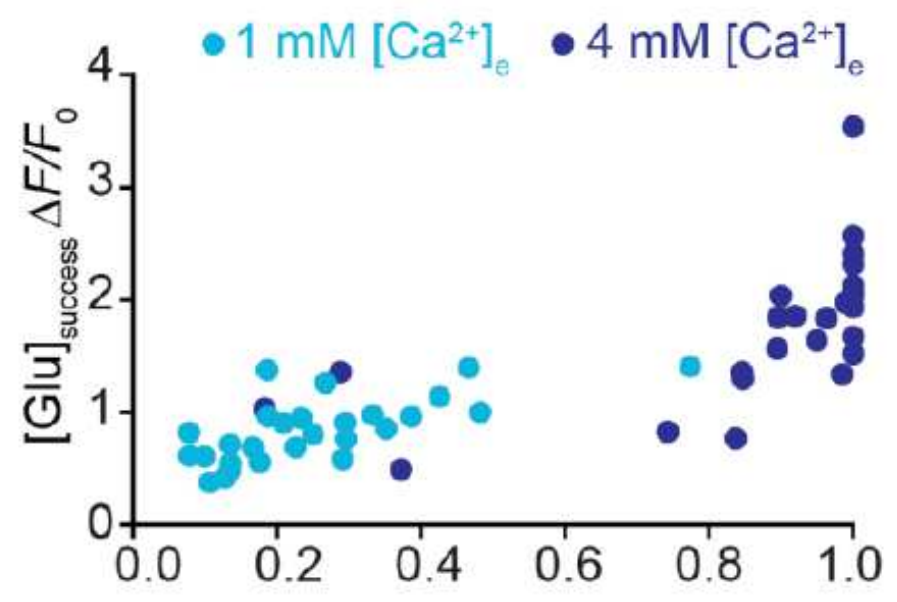

Synaptic release probability b
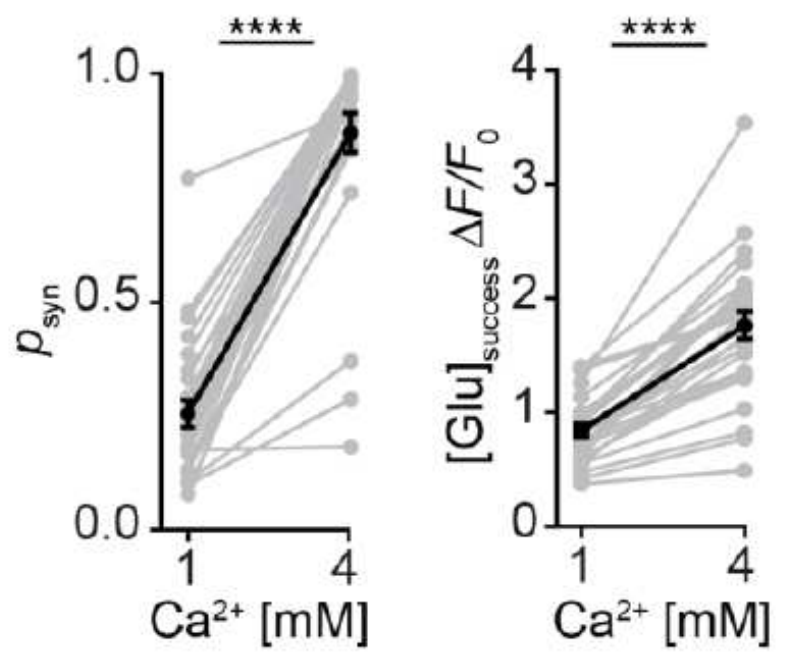

d

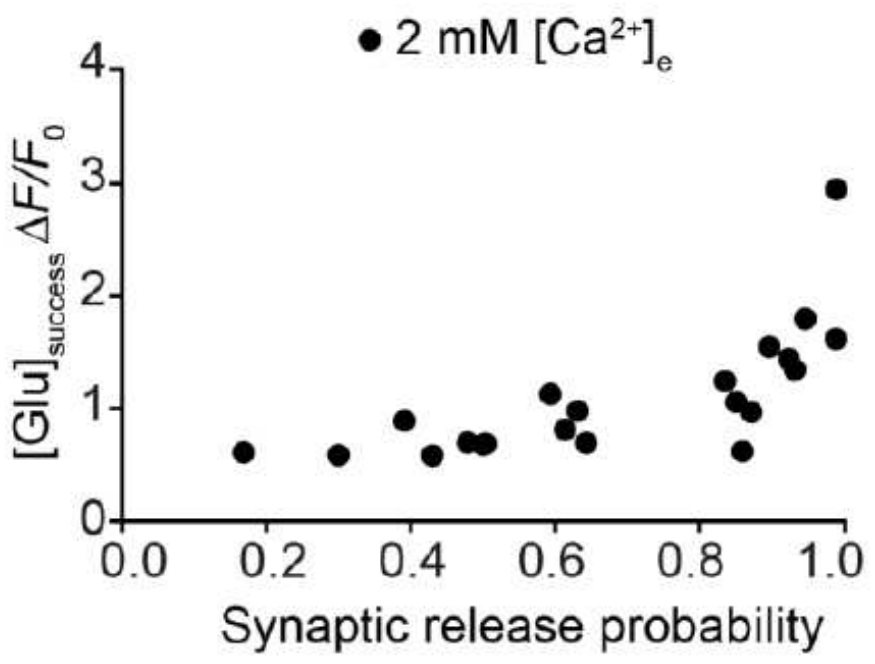

Figure 2

Increasing release probability increases the glutamate concentration of synaptic transmission events. a) Glutamate transients in a single bouton, switching from ACSF containing $1 \mathrm{mM}[\mathrm{Ca} 2+]$ e to $4 \mathrm{mM}[\mathrm{Ca} 2+]$. The light blue and the dark blue lines represent the average of the amplitude of successes in $1 \mathrm{mM}$ [Ca2+]e and $4 \mathrm{mM}[\mathrm{Ca} 2+]$ e respectively. b) Summary of all experiments in $1 \mathrm{mM} / 4 \mathrm{mM}$ [Ca2+]e. The probability of successful glutamate release (left panel) increased from $0.26 \pm 0.03$ in $1 \mathrm{mM}$ [Ca2+] to $0.87 \pm 0.04$ in $4 \mathrm{mM}$ [Ca2+]e (Wilcoxon signed rank test, $\mathrm{p}<0.0001, \mathrm{n}=27$ boutons in 23 slices). The amplitude of fluorescence transients in trials classified as 'success' (right panel) increased from $0.84 \pm$ $0.056 \Delta \mathrm{F} / \mathrm{F} 0$ in $1 \mathrm{mM}[\mathrm{Ca} 2+]$ e to $1.76 \pm 0.12 \Delta \mathrm{F} / \mathrm{F} 0$ in $4 \mathrm{mM}[\mathrm{Ca} 2+]$ e (paired t test, $\mathrm{p}<0.0001, \mathrm{n}=27$ boutons in 23 slices) indicating higher glutamate concentrations in the synaptic cleft under conditions of higher release probability. Values are given as mean \pm SEM. c) The probability of successes was 
correlated with the amplitude of success trials in a non-linear fashion. d) In $2 \mathrm{mM}$ [Ca2+]e, the synaptic release probability of individual synapses ranged from 0.17 to 0.99 . The amplitude of success trials was similar for boutons with low synaptic release probability but rose steeply for psyn $>0.8$.

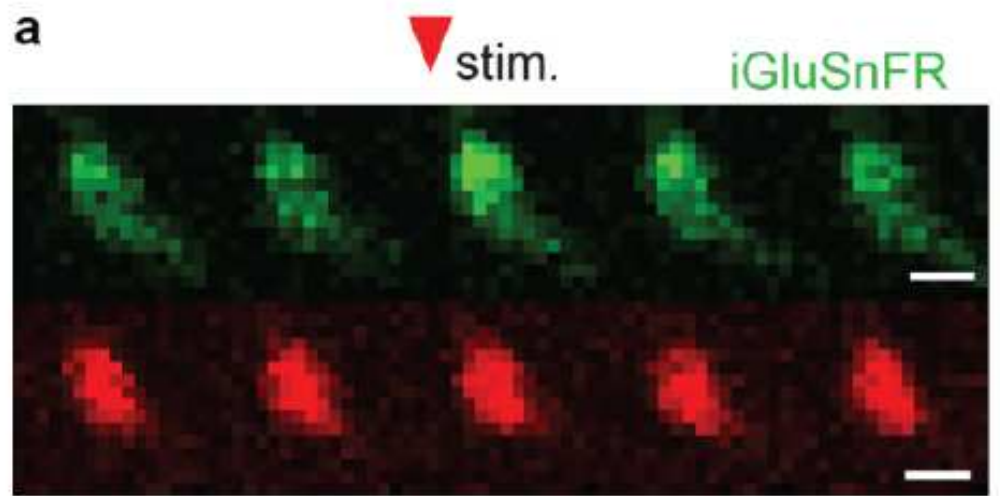

tdimer-2

b

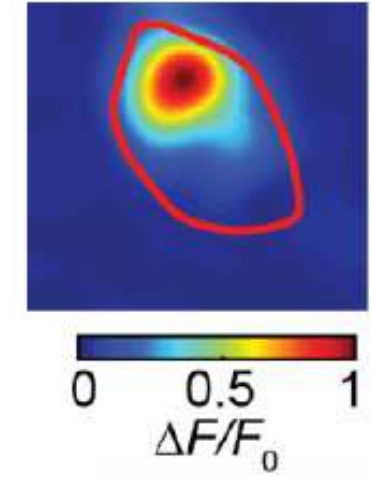

d

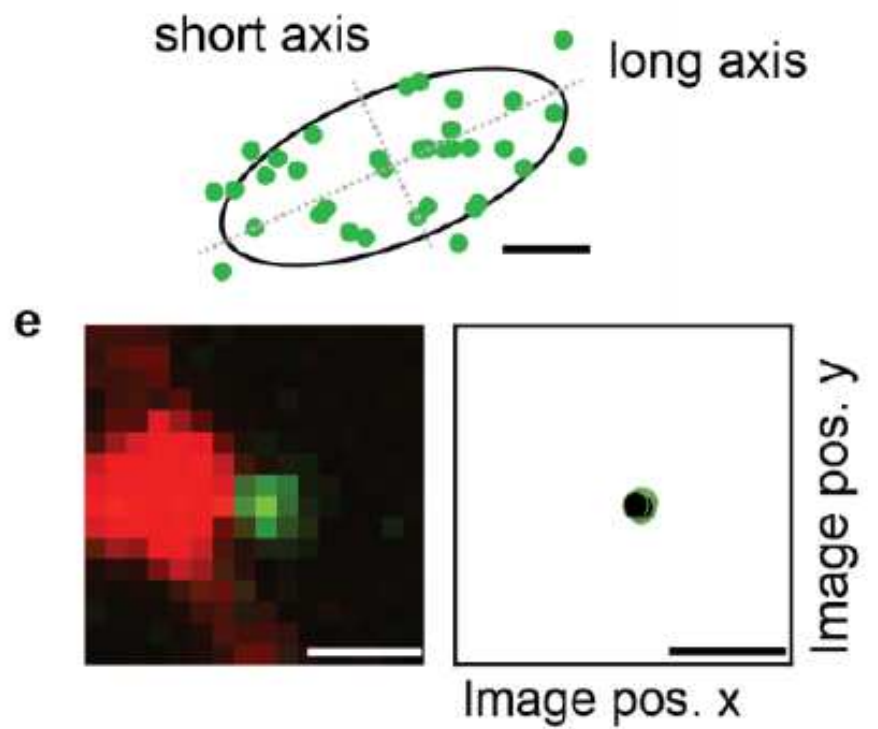

$\mathbf{f}$
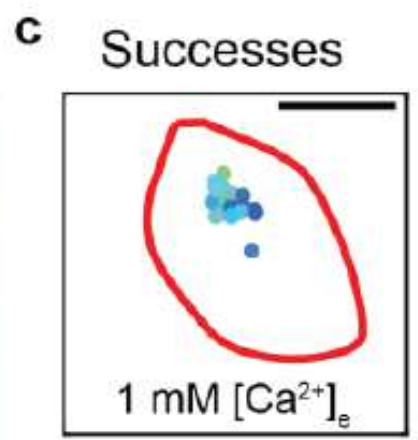

Successes

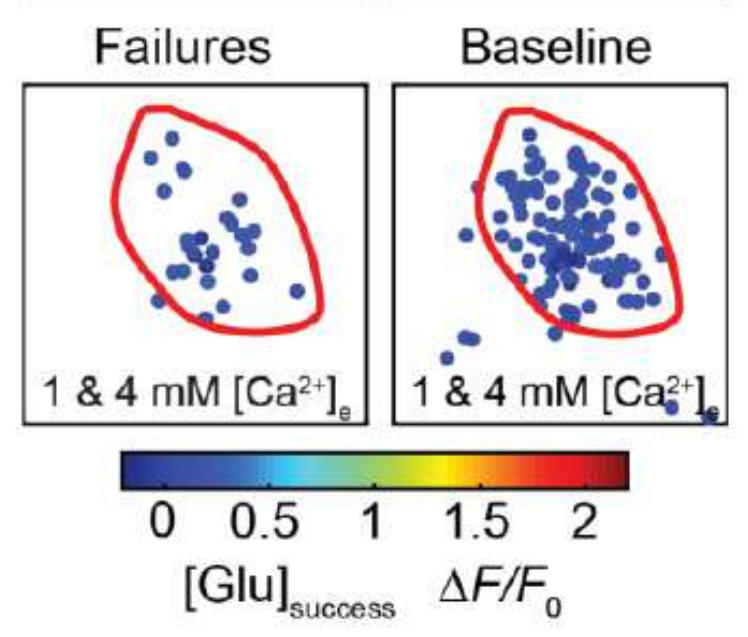

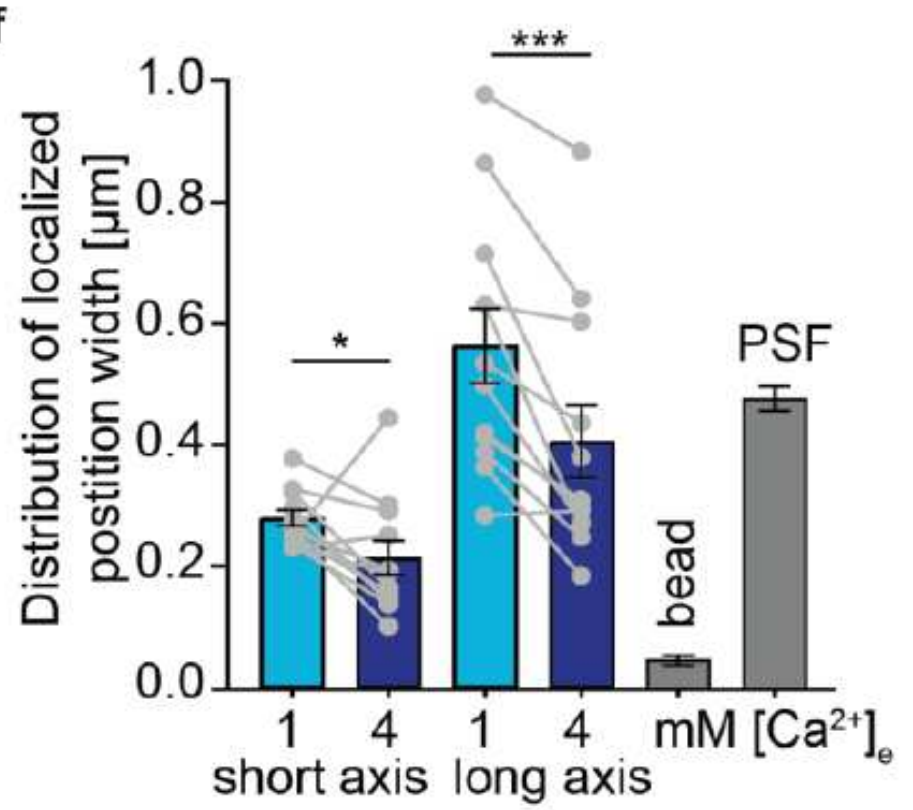

Figure 3

Localizing the fusion site. a) Time-lapse (frame rate $62.5 \mathrm{~Hz}$ ) of a bouton releasing glutamate in response to an individual AP. Scale bar, $1 \mu \mathrm{m}$. b) (Left) top view of average responses of signals classified as successes. (Right) side view of the resulting 2D-Gaussian kernel fits. c) (Upper left) center 
positions of 2D-Gaussian kernel fits in success trials in ACSF containing $1 \mathrm{mM}$ [Ca2+]e. (Upper right) center positions of 2D-Gaussian kernel fit in success trials in ACSF containing $4 \mathrm{mM}$ [Ca2+]e. (Lower left) center positions of 2D-Gaussian kernel fits in failure trials (below $2 \sigma$ of the baseline). (Lower right) center positions of 2D-Gaussian kernel fits in failure frames before the stimulation in ACSF containing $1 \mathrm{mM}$ [Ca2+]e and ACSF containing $4 \mathrm{mM}[\mathrm{Ca} 2+]$ e. Scale bar, $1 \mu \mathrm{m}$. d) Example of a fitted ellipse including 95\% of localized successes (green dots) from a single bouton. The length of the short and long axis of the fitted ellipse of successes measured in $1 \mathrm{mM}[\mathrm{Ca} 2+]$ e and $4 \mathrm{mM}[\mathrm{Ca} 2+]$ e are used to test for a relative enlarging of the release area in $4 \mathrm{mM}[\mathrm{Ca} 2+]$ indicative of a multisynapse bouton. Scale bar, $128 \mathrm{~nm} . \mathrm{e}$ ) (Left) single plane of a two-photon microscope image of a bouton (tdimer2) next to a yellow-green fluorescent microspheric bead $(0.17 \mu \mathrm{m})$. (Right) center positions of 2D-Gaussian kernel fits to the fluorescent signal of a bead. 50 consecutive images were acquired at $62.5 \mathrm{~Hz}$. Scale bars, $1 \mu \mathrm{m}$. f) Histogram of short and long axis of the fitted ellipse to the localized responses and FWHM distribution of the localized bead and PSF measurement. Success localization short axis, $0.28 \pm 0.01 \mu \mathrm{m}$ in $1 \mathrm{mM}$ [Ca2+]e and $0.21 \pm 0.03 \mu \mathrm{m}$ in $4 \mathrm{mM}[\mathrm{Ca} 2+]$ (Wilcoxon test, $\mathrm{p}=0.04, \mathrm{n}=12$ boutons); success localization long axis, $0.56 \pm 0.06 \mu \mathrm{m}$ in $1 \mathrm{mM}$ [Ca2+] and $0.41 \pm 0.06 \mu \mathrm{m}$ in $4 \mathrm{mM}$ [Ca2+]e (Wilcoxon test, $p=0.001, n=12$ boutons); bead localization $0.05 \pm 0.01 \mu \mathrm{m}$; PSF bead, $0.48 \pm 0.02 \mu \mathrm{m})$. Values are given as mean \pm SEM. 

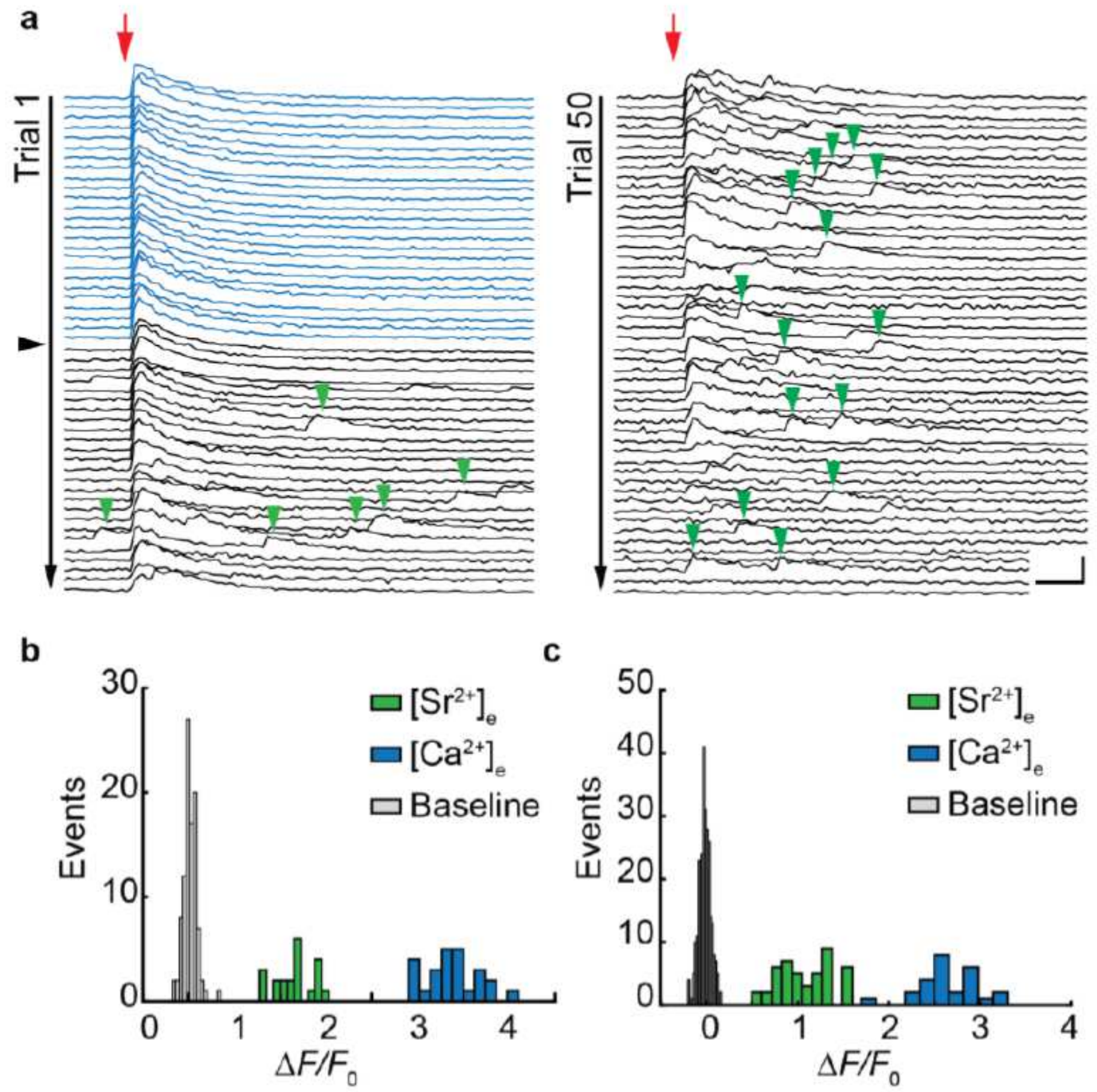

\section{Figure 4}

Desynchronized release events reveal quantal size. a) iGluSnFR changes in fluorescence (single bouton) in response to single APs in ACSF containing $4 \mathrm{mM}$ [Ca2+]e (blue traces). Red arrow indicates stimulation onset. Synaptic release probability was 1 (no failures). The black arrowhead indicates the start of $4 \mathrm{mM}$ [Sr2+]e. wash-in. During wash-in of $4 \mathrm{mM}[\mathrm{Sr} 2+]$ e discrete events appear in the wake of the stimulation (green arrowheads). Scale bar, $2 \Delta \mathrm{F} / \mathrm{F} 0$ and $20 \mathrm{~ms}$. b) Amplitude histogram of evoked responses measured in $4 \mathrm{mM}$ [Ca2+]e (blue bars) and delayed events measured in $4 \mathrm{mM}$ [Sr2+]e (green bars) and fluctuation of baseline fluorescence (gray bars) of the example shown in (a). c) Amplitude histograms of 
a bouton with evoked responses measured in $4 \mathrm{mM}$ [Ca2+]e (blue bars), delayed events measured in 4 $\mathrm{mM}$ [Sr2+]e (green bars) and fluctuation of baseline fluorescence (gray bars). The bin size is smaller for the baseline fluctuations for display purposes.

a

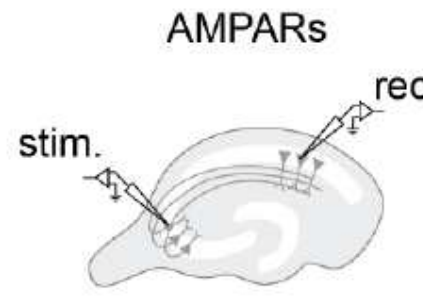

C

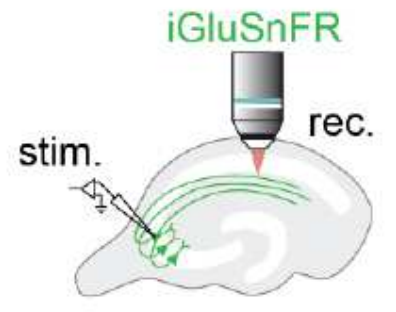

rec.
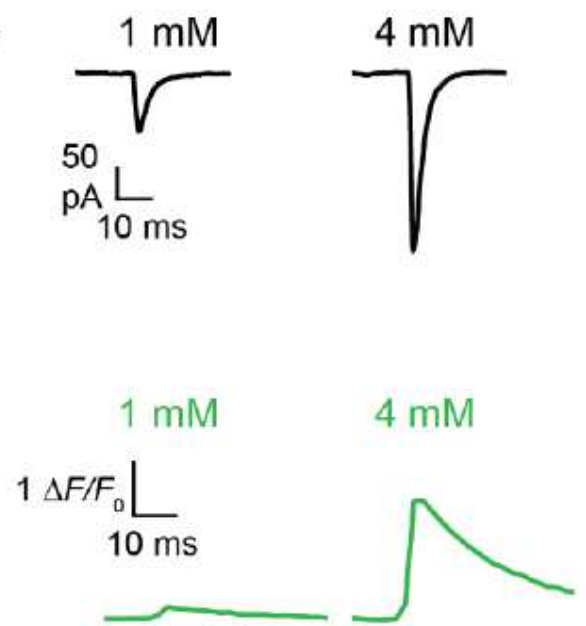

b

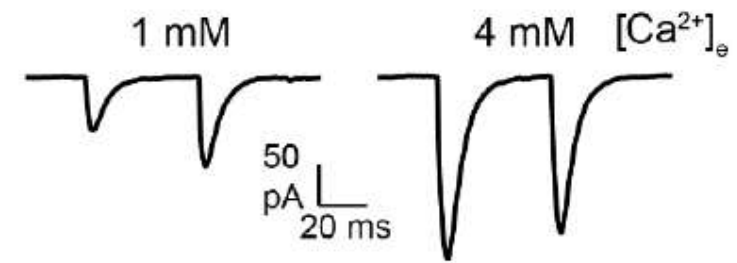

d

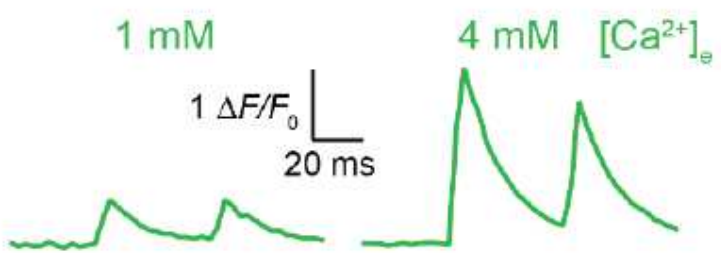

e

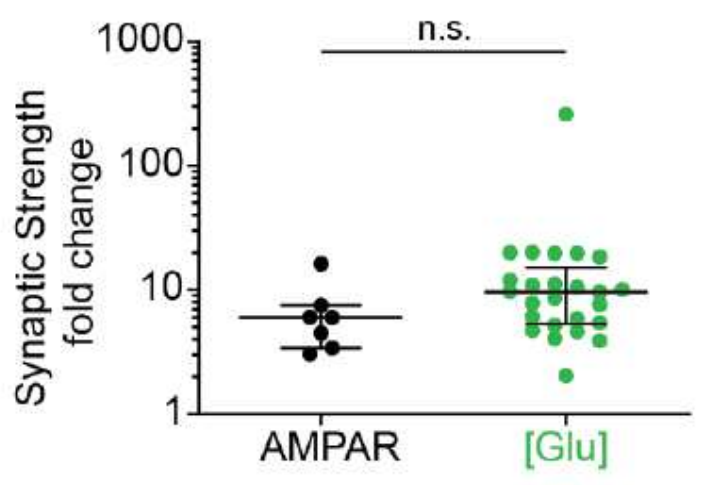

f

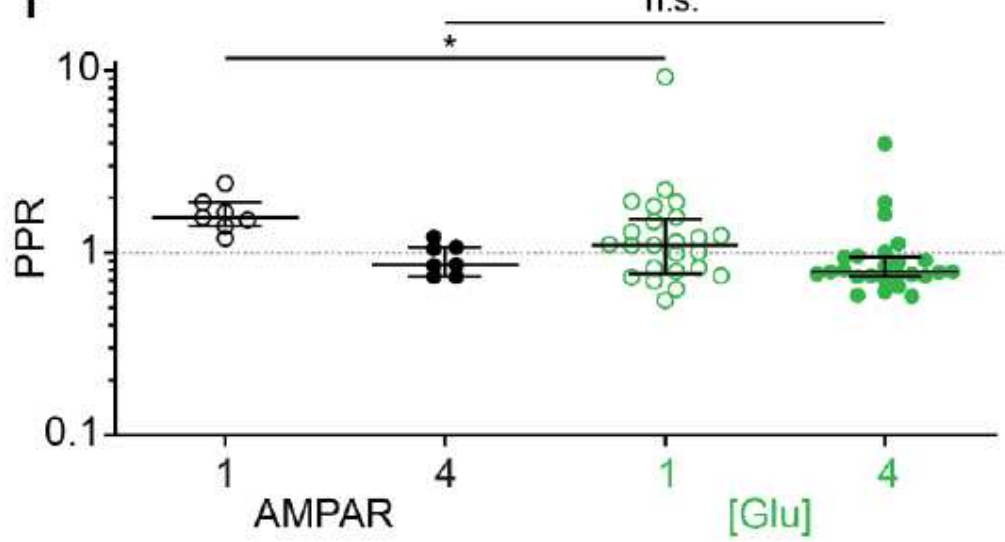

\section{Figure 5}

Multivesicular release increases the gain and the signal to noise ratio of synapses. a) EPSCs were measured by dual patch-clamp recordings from connected CA3-CA1 pyramidal cell pairs. Perfusion was switched from $1 \mathrm{mM}$ to $4 \mathrm{mM}$ [Ca2+]e, leading to increased EPSC amplitudes. Traces show EPSCs (average of $\sim 40$ trials) from the CA1 pyramidal cell. b) Representative EPSCs (average of $\sim 40$ trials) in response to the presynaptic paired-pulse stimulation (ISI $48 \mathrm{~ms}$ ) recorded in $1 \mathrm{mM}$ and $4 \mathrm{mM}$ [Ca2+]e. c) iGluSnFR signals were measured as described previously (Fig. 1). Traces show the evoked change in fluorescence (average of $\sim 60$ trials) from a single bouton in CA1 stratum radiatum. The switching sequence (low - high [Ca2+]e / high - low [Ca2+]e) was reversed between experiments. d) Representative iGluSnFR signals (average of $\sim 60$ trials) from a single bouton in CA1 stratum radiatum in response to the paired-pulse stimulation. e) Increasing [Ca2+] from 1 to $4 \mathrm{mM}$ increased the median amplitude of AMPAR-mediated EPSCs by a factor of 6.02, interquartile range (IQR): $3.4-7.5$ fold, $n=7$ pairs, the median iGluSnFR response by 9.63 fold, IQR: $5.3-15.2$ fold, $n=25$ boutons. There is no significant 
difference between the fold change in AMPARs and iGluSnFR responses (Mann-Whitney, $p=0.0832$ ). $f$ ) EPSCs showed paired-pulse facilitation in $1 \mathrm{mM}$ [Ca2+]e, (median PPR: 156\%, IQR: $1.4 \%-1.9 \%, \mathrm{n}=7$ pairs) and paired-pulse depression in $4 \mathrm{mM}$ [Ca2+]e (median PPR: 86\%, IQR: 74\%-107\%, $\mathrm{n}=7$ pairs). iGluSnFR responses showed paired-pulse facilitation in $1 \mathrm{mM}$ [Ca2+]e (median PPR: 110\%, IQR: 77\%$152 \%, \mathrm{n}=25$ boutons) and paired-pulse depression in $4 \mathrm{mM}$ [Ca2+]e (median PPR: 79\%, IQR: 74\%-95\%, $\mathrm{n}$ $=25$ boutons). There is a significant difference between the AMPARs PPR and the iGluSnFR PPR in $1 \mathrm{mM}$ [Ca2+]e (Mann-Whitney test, $p=0.03$ ). However, there is no significant difference between the AMPARs PPR and the iGluSnFR PPR in $4 \mathrm{mM}$ [Ca2+]e (Mann-Whitney test, $p=0.37$ ). Value with a PPR $=0$ is not plotted for display purpose but was used for statistics. Values are plotted as median with IQR. 
a
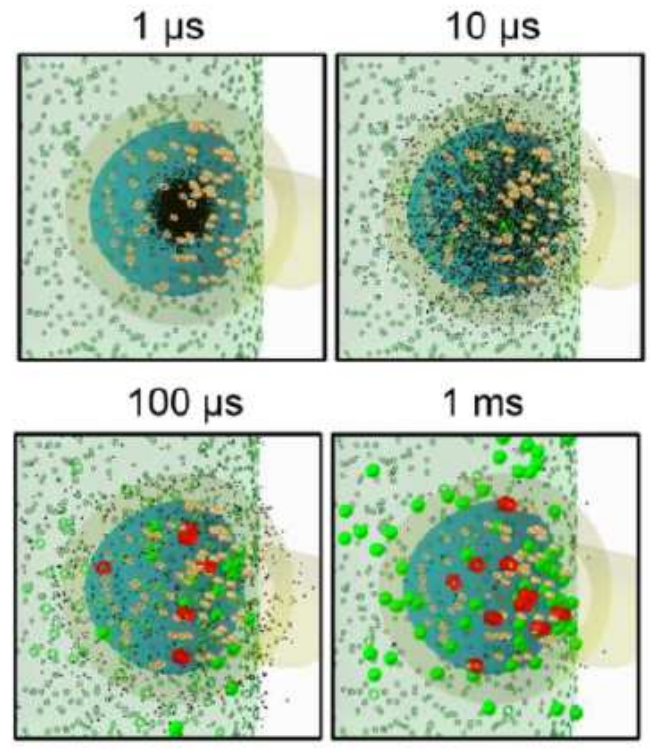

- glutamate oiGluSnFR

o iGluSnFR-glu dim iGluSnFR-glu $_{\text {bright }}$ - AMPAR oopen AMPARs

C
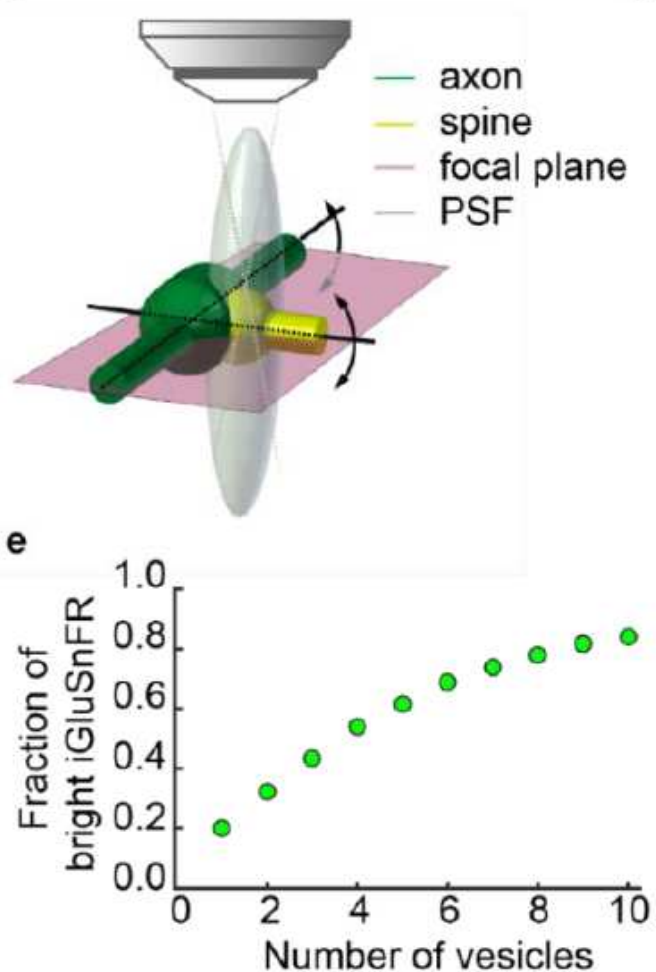

d b
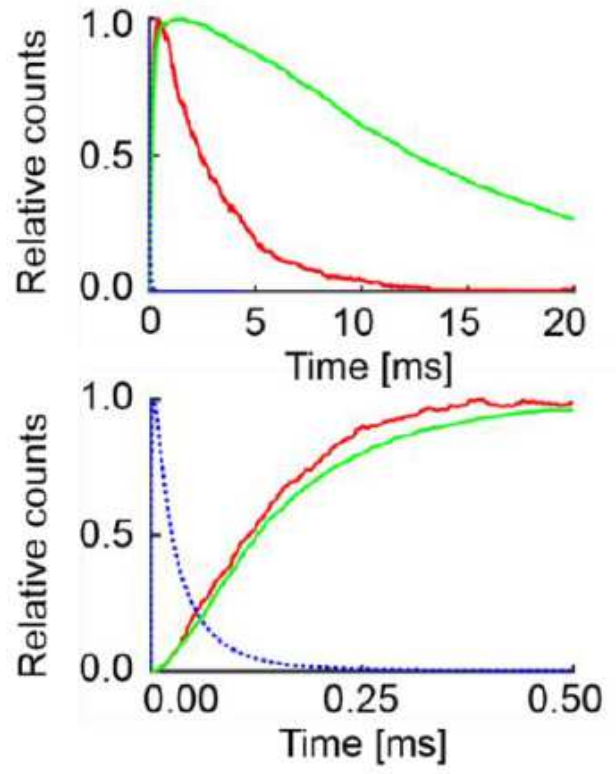

.... glutamate - iGluSnFR-glu bright - open AMPARs
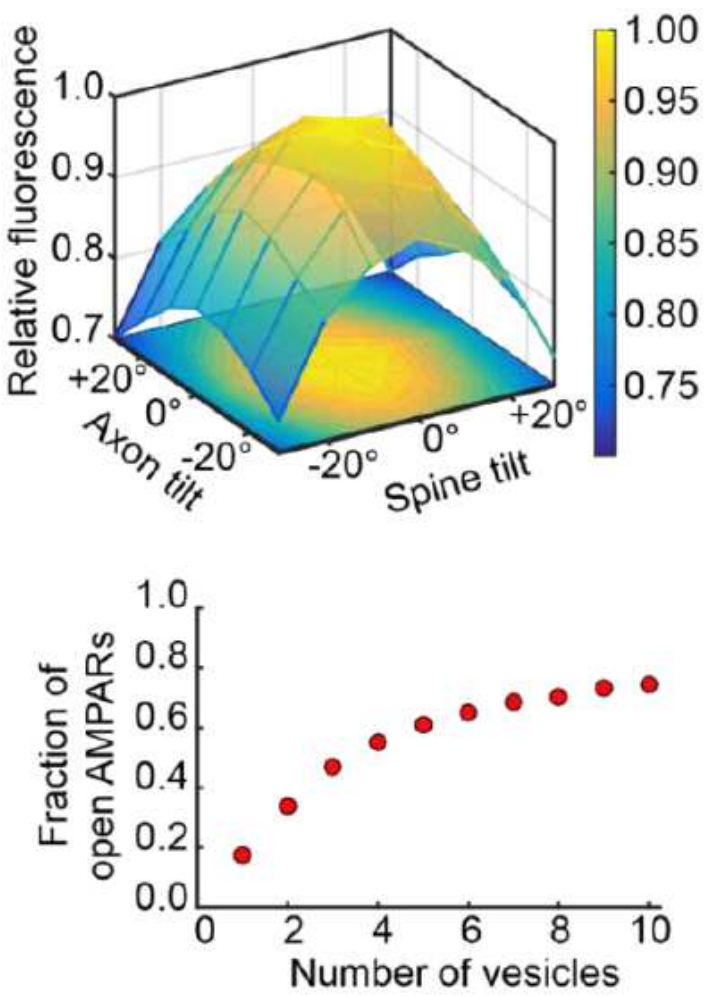

\section{Figure 6}

Monte-Carlo-simulation of glutamate diffusion in the synaptic cleft. a) Simulated glutamate dynamics within the synaptic cleft upon vesicle release. The model consists of a presynaptic terminal with iGluSnFR molecules opposed to a spine with AMPARs randomly distributed in a disc of $300 \mathrm{~nm}$, separated by a $20 \mathrm{~nm}$ synaptic cleft. The synapse is embedded in a network of astrocytes equipped with glutamate transporters. b) Simulated reaction time profile of AMPARs opening and iGluSnFR in a bright 
fluorescent state upon release of a single vesicle containing 3000 molecules of glutamate (upper panel) and close-up view of the kinetics from 0 to $50 \mathrm{~ms}$ (lower panel). c) Simulated fluorescence measurements under different orientations of axon and spine with respect to the optical axis. iGluSnFR fluorescence was evaluated inside the PSF. Tilting the axon or the spine reduces $\triangle F / F 0$, as more iGluSnFR molecules outside the synaptic cleft fall within the PSF, contributing to the resting fluorescence F0. d) Normalized fluorescence transient with respect to tilted positions of axon or spine. e) Fraction of bright iGluSnFR bound to glutamate (left panel) and fraction of open AMPARs (right panel) after release of 1-10 vesicles (3000 glutamate molecules per vesicle). Occupancy of iGluSnFR and AMPA receptors is similar after a single vesicle release, resulting in similar saturation curves. 
a

step

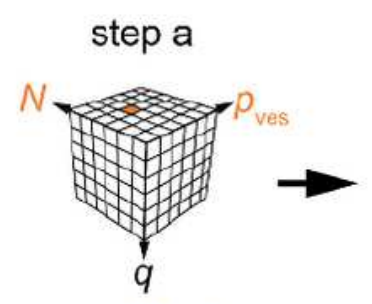

step b

Photon shot

noise

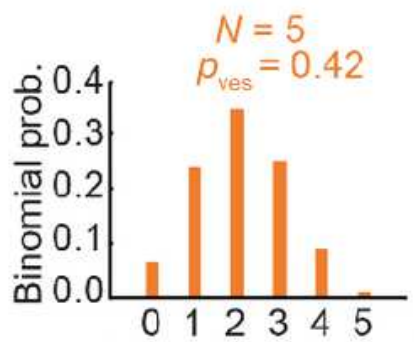

\# vesicles released
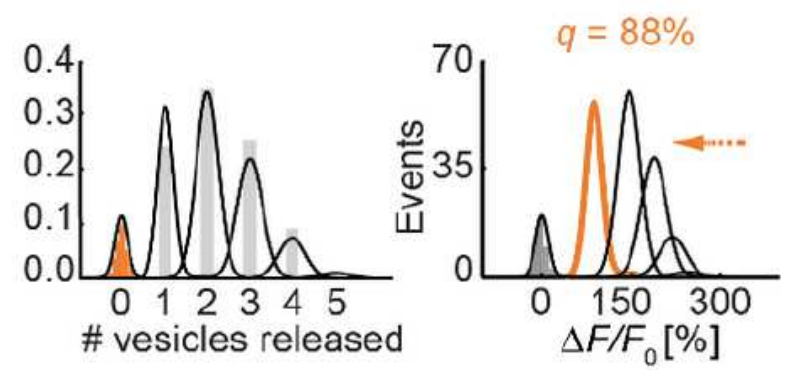

step c

step d

\# vesicles released
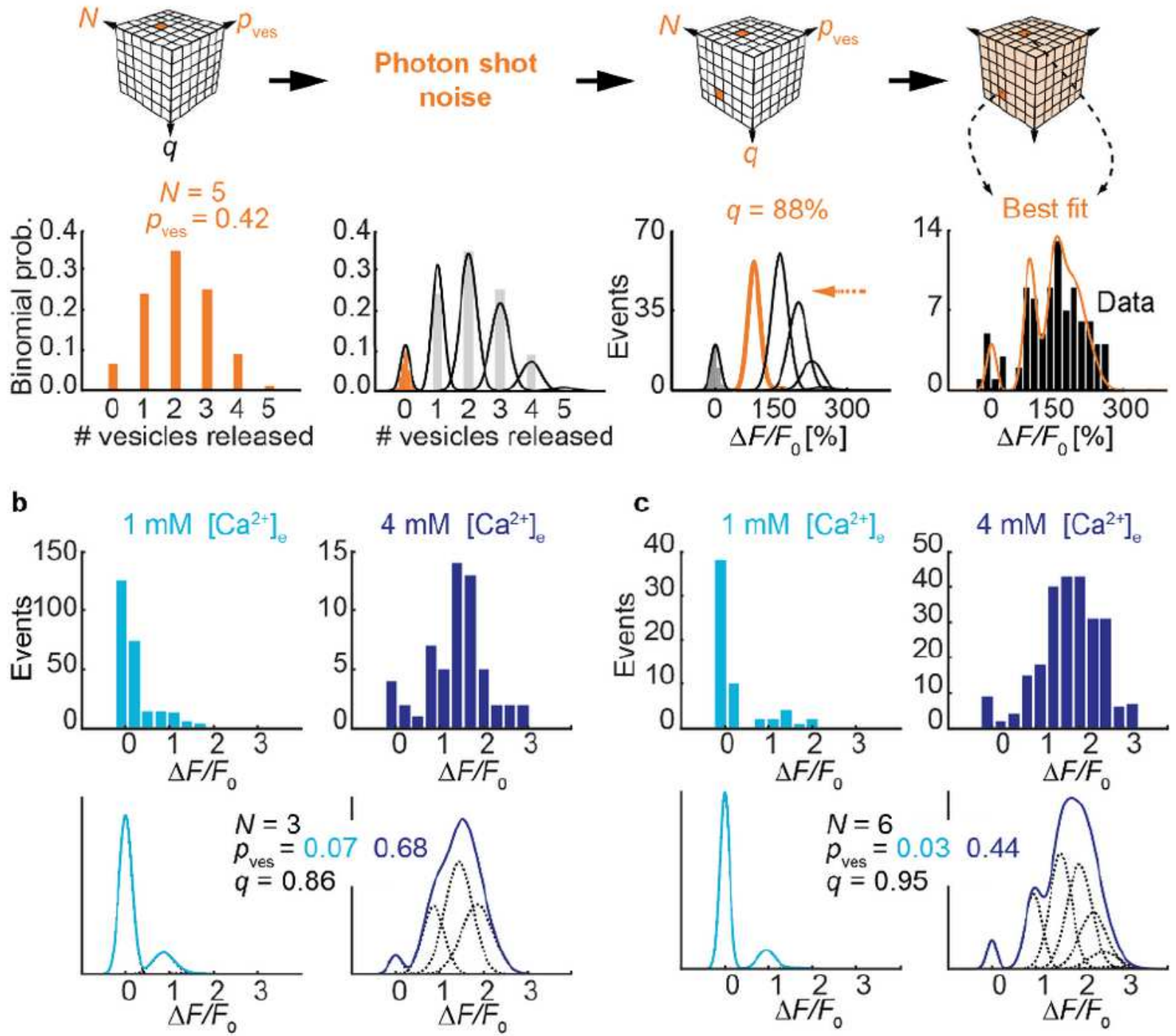

d
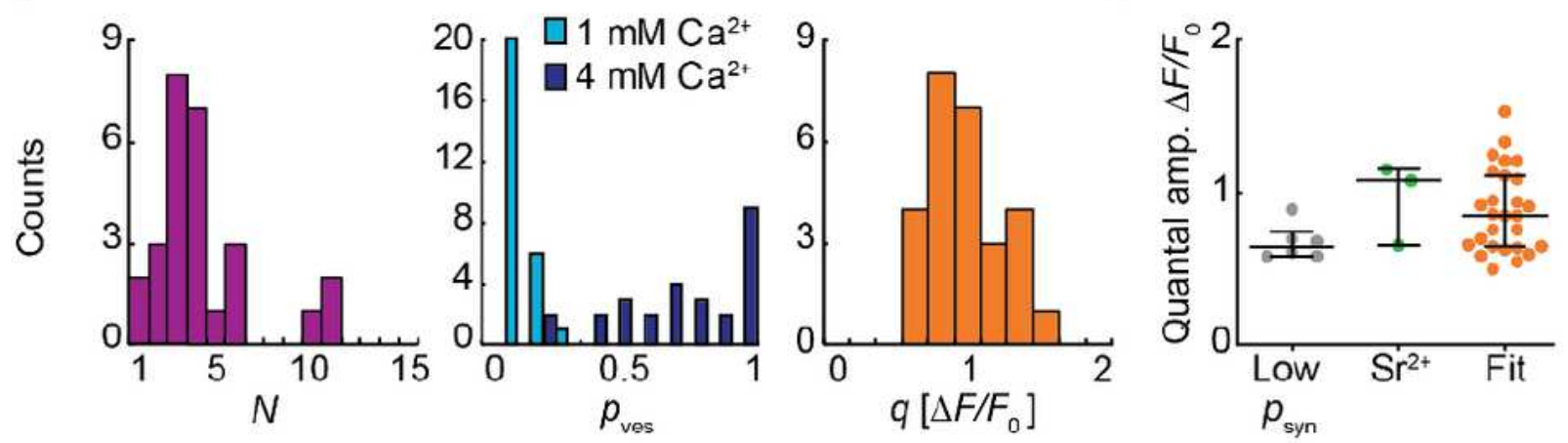

Figure 7

Extracting N, pves and q of Schaffer collateral synapses using a binomial model. a) Schematic view of the exhaustive search of the quantal parameters $\mathrm{N}$, pves and q. For a combination of $\mathrm{N}$ and pves, the binomial probabilities of the possible outcomes were calculated (here: $\mathrm{N}=5$ vesicles and pves $=0.42$ ). Without stimulation, amplitudes were normally distributed around zero (orange bars). The width of this noise distribution, a function of the number of photons detected from a particular bouton, was used to 
determine the width of the expected Gaussian probability density functions. A chosen quantal amplitude $\mathrm{q}$ (here: $88 \% \triangle \mathrm{F} / \mathrm{FO}$ ) sets the spacing of the Gaussian probability density functions. iGluSnFR saturation (max. $\triangle F / F 0=440 \%$ ) was considered when setting the expected amplitudes of multi-quantal events, leading to a compression (orange arrow) of the individual Gaussians. The resulting probability density function (sum of the Gaussians) was compared to the measured amplitude distribution of a single bouton (black bars, recorded in $2 \mathrm{mM}[\mathrm{Ca} 2+] \mathrm{e}$ ). The root mean square (RMS) error was calculated and the best fit (shown here) was selected to determine the synaptic parameters. b-c) Single bouton response distributions recorded in $1 \mathrm{mM}[\mathrm{Ca} 2+]$ e and $4 \mathrm{mM}$ [Ca2+]e. Two examples from two different slice cultures. The binomial fitting procedure was applied to both histograms, searching for the best combined fit under the condition that $\mathrm{N}$ and $\mathrm{q}$ had to be identical in $1 \mathrm{mM}[\mathrm{Ca} 2+]$ e and $4 \mathrm{mM}$ [Ca2+]e while pves could be different. Best fit probability density functions and extracted parameters are shown below the experimental data. d) Extracted quantal parameters, $\mathrm{N}$ median: 4 vesicles, IQR: 3-5 vesicles; pves in $1 \mathrm{mM}$ [Ca2+] median: 0.07, IQR: 0.03-0.11; pves in $4 \mathrm{mM}$ [Ca2+] e median: 0.71, IQR = 0.45-0.96; q median = $0.85 \Delta F / F 0$, IQR: 0.65-1.11 $\Delta F / F 0, n=27$ boutons. The numbers delimit the right edge of their respective bin. e) Estimating quantal amplitude by three different approaches using independent datasets.

Measurements report no significant difference (Kruskal-Wallis test, $p=0.13$ ) between the quantal sizes. psyn < 0.5 boutons in $2 \mathrm{mM}$ [Ca2+]e median: $0.65 \Delta \mathrm{F} / \mathrm{F} 0$, IQR: 0.59-0.75 $\Delta \mathrm{F} / \mathrm{F} 0, \mathrm{n}=6$ boutons; delayed events in $4 \mathrm{mM}$ [Sr2+]e wash-in experiments median: 1.08 $\Delta \mathrm{F} / \mathrm{FO}, \mathrm{IQR}: 0.66-1.15 \Delta \mathrm{F} / \mathrm{F} 0, \mathrm{n}=3$ boutons; binomial fitting procedure median: $0.85 \Delta \mathrm{F} / \mathrm{F} 0, \mathrm{IQR}: 0.65-1.11 \Delta \mathrm{F} / \mathrm{F} 0, \mathrm{n}=27$ boutons. Values are given as median with IQR.
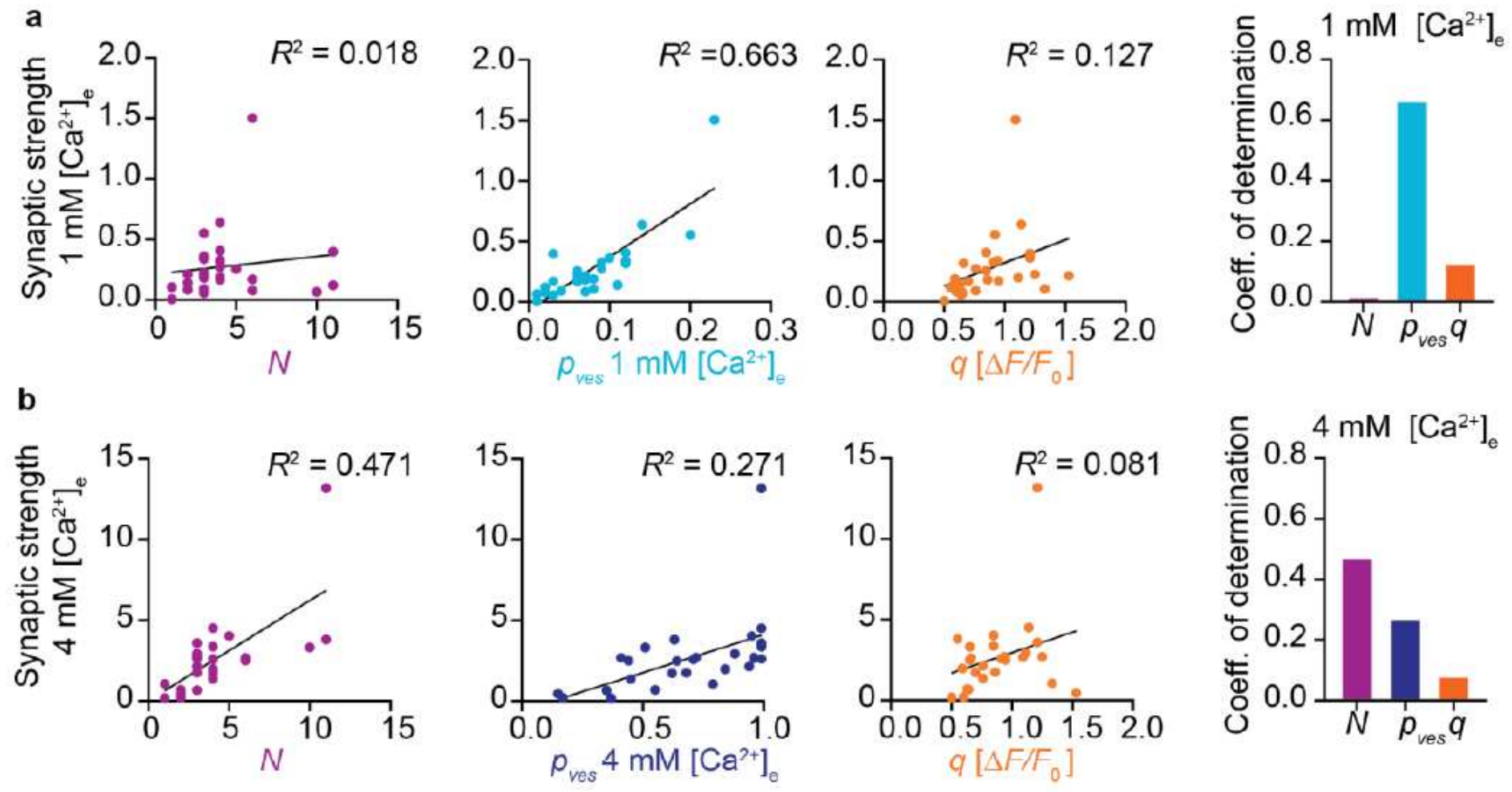

\section{Figure 8}


Quantal parameters and synaptic strength. a) Correlations between the calculated synaptic strength in 1 $\mathrm{mM}[\mathrm{Ca} 2+]$ e and the extracted quantal parameters $\mathrm{N}$, pves and $\mathrm{q}(\mathrm{n}=27$ boutons). b) Correlations between the calculated synaptic strength in $4 \mathrm{mM}[\mathrm{Ca} 2+]$ e and the extracted quantal parameters $\mathrm{N}$, pves and $q$ ( $n=27$ boutons). (First 3 panels) Plot of the calculated synaptic strength in $4 \mathrm{mM}$ [Ca2+]e from the extracted quantal parameters as shown in Fig. 7 (product of N, pves and q) as a function of N, pves $4 \mathrm{mM}$ [Ca2+]e and $q(n=27$ boutons). (Right panel) summary of the coefficient of determination for the linear correlations between synaptic strength in $4 \mathrm{mM}[\mathrm{Ca} 2+]$ e and $\mathrm{N}(\mathrm{R} 2=0.471)$, pves in $1 \mathrm{mM}[\mathrm{Ca} 2+] \mathrm{e}(\mathrm{R} 2=$ $0.271)$ and $q(R 2=0.081)$.

\section{Supplementary Files}

This is a list of supplementary files associated with this preprint. Click to download.

- ExtendedDataNatNeurosci.pdf 